ARTICLE

Received 10 Oct 2013 | Accepted 2 Apr 2014 | Published 7 May 2014

DOI: $10.1038 /$ ncomms 4790

OPEN

\title{
Glycinergic inhibition tunes coincidence detection in the auditory brainstem
}

Michael H. Myoga ${ }^{1}$, Simon Lehnert ${ }^{1}$, Christian Leibold ${ }^{1}$, Felix Felmy ${ }^{1,2, \star} \&$ Benedikt Grothe ${ }^{1, \star}$

Neurons in the medial superior olive (MSO) detect microsecond differences in the arrival time of sounds between the ears (interaural time differences or ITDs), a crucial binaural cue for sound localization. Synaptic inhibition has been implicated in tuning ITD sensitivity, but the cellular mechanisms underlying its influence on coincidence detection are debated. Here we determine the impact of inhibition on coincidence detection in adult Mongolian gerbil MSO brain slices by testing precise temporal integration of measured synaptic responses using conductance-clamp. We find that inhibition dynamically shifts the peak timing of excitation, depending on its relative arrival time, which in turn modulates the timing of best coincidence detection. Inhibitory control of coincidence detection timing is consistent with the diversity of ITD functions observed in vivo and is robust under physiologically relevant conditions. Our results provide strong evidence that temporal interactions between excitation and inhibition on microsecond timescales are critical for binaural processing.

\footnotetext{
${ }^{1}$ Division of Neurobiology, Department Biologie II, Ludwig-Maximilians-Universität München, Großhaderner Straße 2, Planegg-Martinsried D-82152, Germany. ${ }^{2}$ Biolmaging Zentrum, Ludwig-Maximilians-Universität München, Großhaderner Straße 2, Planegg-Martinsried D-82152, Germany. ${ }^{\star}$ These authors contributed equally to this work. Correspondence and requests for materials should be addressed to B.G. (email: grothe@lmu.de).
} 
C oincidence detection of afferent synaptic inputs is a hallmark of neuronal processing. In many brain areas, such as the cerebral cortex and cerebellum, neurons integrate hundreds of synaptic inputs and perform coincidence detection with millisecond resolution. In contrast, neurons in the mammalian brainstem nucleus of the medial superior olive (MSO) integrate relatively few, clearly defined synaptic inputs ${ }^{1}$, but must perform coincidence detection with orders of magnitude higher precision. They detect microsecond differences in the arrival time of low-frequency sounds between the ears (interaural time differences or ITDs) ${ }^{2}$ and generate a rate code that represents the location of sounds in the horizontal plane ${ }^{3}$.

MSO neurons generally respond best to contralateral-leading ITDs $^{4-8}$. To allow best coincidence detection of such temporally staggered inputs, the circuit must generate internal delays to compensate for the external delay created by the head. A longer excitatory path length from the contralateral side can explain this phenomenon at the population level, but there is increasing evidence that axonal length disparity ${ }^{9}$ alone is insufficient to explain the diversity of preferred ITDs observed in vivo ${ }^{10-13}$. Thus, it has become a crucial question how individual neurons within the population are tuned to their specific ITDs ${ }^{7,14-18}$, an answer to which is necessary for a complete understanding of low-frequency sound localization in mammals.

Previous studies found that blocking glycinergic inhibition in vivo shifted the peak of ITD functions, providing direct evidence that inhibition is important for ITD tuning 6,7 . As glycinergic inputs to the MSO are extremely fast and precise ${ }^{1,6,19-23}$, a computational model was developed in which the relative arrival time of excitatory and inhibitory inputs tunes neurons to their preferred ITDs by modulating the peak timing of the summated input from each side $7,17,24,25$. However, this model was not rigorously tested under naturally occurring conditions and has been recently challenged ${ }^{8,26}$. In one study, the difference in arrival time between inhibition and excitation was measured in vitro. Inhibition was indeed precise and actually preceded excitation, but the average timing condition did not tune ITD functions, and it was concluded that inhibition was not important for ITD tuning ${ }^{26}$. In a second study, ITD functions were determined by juxtacellular and whole-cell recordings in vivo, but preceding inhibition as measured in vitro could not be detected $^{8}$. Thus, there are now discrepancies between the existing data ${ }^{6-8,24-26}$ and confusion regarding the relevance of inhibition for ITD tuning $3,27-29$.

To understand these discrepancies, we systematically reevaluate the role of precise inhibition in synaptic integration and coincidence detection under well-defined, physiologically relevant conditions using acute brain slices from adult Mongolian gerbils. We find that inhibition indeed tunes coincidence detection in a manner consistent with the original model ${ }^{6,7,24,28}$, but much more dynamically than originally proposed. Moreover, we provide evidence that the specific involvement of inhibition in ITD tuning in vivo may not be discernable with currently available techniques. Thus, we expand the framework for the role of inhibition in ITD tuning and provide explanations for the discrepancies in the literature.

\section{Results}

Inhibition modulates the peak timing of excitation. Coincidence detection neurons in the MSO are strikingly bipolar in shape (Fig. 1a) ${ }^{30}$ and receive bilateral excitatory and inhibitory synaptic inputs (Fig. 1b) ${ }^{31}$. Because synaptic kinetics and input resistance change markedly during development ${ }^{20,21,32}$, we restricted our primary experiments exclusively to mature gerbils of ages postnatal day (P) 60-90. As a basis for our quantitative re-evaluation of cellular ITD sensitivity, we first extracted the time course of excitatory and inhibitory synaptic conductances (EPSGs and IPSGs, respectively) in a separate set of voltage-clamp experiments (Supplementary Fig. 1). Individual conductance waveforms were then selected and injected as templates into the soma using conductance-clamp to simulate excitatory and inhibitory postsynaptic potentials (EPSPs and IPSPs, respectively), which produced events that largely resembled synaptically evoked responses (Supplementary Fig. 2). This allowed us to investigate precise temporal interactions between excitation and inhibition based on synaptic responses measured in mature tissue.

A fundamental principle of the original inhibitory model of ITD tuning proposes that inhibition modulates the timing of best coincidence by enforcing a shift in the peak timing of excitation on each side $6,7,24,28$. As there is strong evidence that the arrival times of excitatory and inhibitory inputs to the MSO is exquisitely precise ${ }^{19,33,34}$, we determined whether a precisely timed IPSP can shift the peak timing of an EPSP, here arbitrarily designated to the contralateral side (Fig. 1). Inhibitory inputs are predominantly restricted to the soma ${ }^{35-37}$, and thus simulating IPSPs with conductance-clamp is suitable to mimic realistic IPSPs. However, because excitatory inputs predominantly target the dendrites ${ }^{35}$ we compared the influence of a single conductance-clamp-simulated (G-inject) IPSP on the peak timing of a synaptically evoked (F-stim) EPSP (Fig. 1c) and a conductance-clamp-simulated EPSP (Fig. 1d) in the same recording. We used an IPSG template with a decay time constant $\left(\tau_{\text {IPSG }}\right)$ that represented our population average $(1.5 \mathrm{~ms})$ and an EPSG template with a decay time constant $\left(\tau_{\mathrm{EPSG}}\right)$ that generated an EPSP matching the kinetic profile of the synaptically evoked EPSP $(0.2-0.3 \mathrm{~ms}$, where $0.3 \mathrm{~ms}$ was the average; Supplementary Fig. 2). EPSG and IPSG peak amplitudes were then adjusted to achieve an effective excitation-inhibition (E-I) ratio of 1:1 (see Methods).

Because the actual arrival times of excitation and inhibition to the MSO in vivo is not known, we investigated a broad range of relative inhibitory timing conditions $\left(\Delta t_{\text {inh }}\right.$; Fig. 1e). Indeed we found timing conditions that advanced the peak timing of both the synaptically evoked and conductance-clamp-simulated EPSPs $\left(\Delta t_{\text {inh }}=0.1 \mathrm{~ms}\right.$, F-stim: $-62 \pm 8 \mu \mathrm{s}$; G-clamp: $-53 \pm 3 \mu \mathrm{s}, n=7$; Fig. 1f,g, left) and others that delayed the peak of the EPSPs, albeit to a lesser extent $\left(\Delta t_{\text {inh }}=-0.6 \mathrm{~ms}\right.$, F-stim: $21 \pm 9 \mu \mathrm{s}$; G-clamp: $4 \pm 6 \mu \mathrm{s}, n=7$; Fig. 1f,g, right). The similarity of the timingdependent peak shifts between synaptically evoked and conductance-clamp-simulated EPSPs $\left(\Delta t_{\text {inh }}=0.1 \mathrm{~ms}: \quad P=0.986\right.$; $\Delta t_{\text {inh }}=-0.6 \mathrm{~ms}: \quad P=0.647$, two-way analysis of variance (ANOVA)) indicates that inhibition influences synaptic integration at the soma (Fig. 1h). This was further supported by separate dual-electrode conductance-clamp experiments that revealed no substantial difference in PSP kinetics or the magnitude of inhibition-enforced EPSP peak shifts compared with singleelectrode experiments (Supplementary Fig. 3). Taken together, these findings demonstrate that single-electrode conductanceclamp is suitable to investigate precise temporal interactions between excitation and inhibition in MSO neurons. Thus, inhibition can indeed modulate the peak timing of excitation from either side in a bidirectional manner depending on its arrival time relative to excitation. These findings are consistent with the basic predictions of the original inhibitory model $\mathrm{l}^{6,7,24,28}$ as well as recent reports ${ }^{26}$

Input speed and balance influence peak shift magnitude. Conductance-clamp is advantageous over synaptic stimulation in that many parameters can be tested quickly in a single recording. 
a

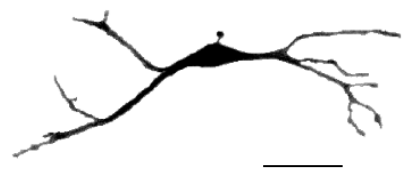

c

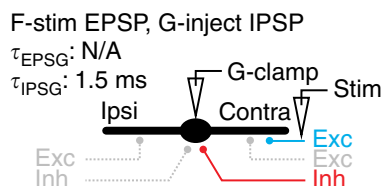

f

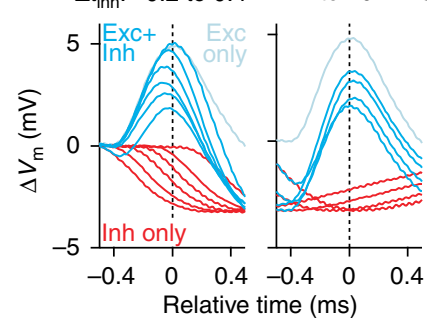

b

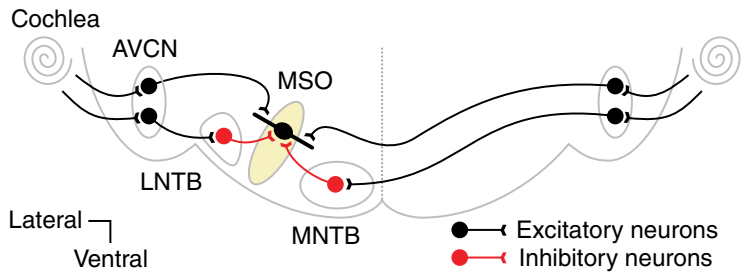

d

G-inject EPSP and IPSP

$\tau_{\text {EPSG }}: 0.2-0.3 \mathrm{~ms}$

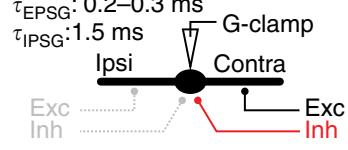

e

Timing convention

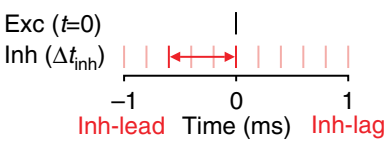

g

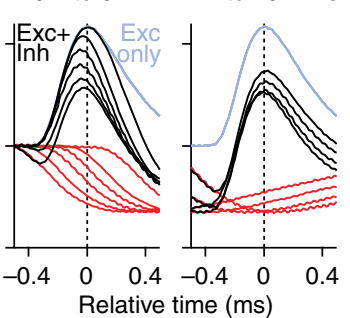

h

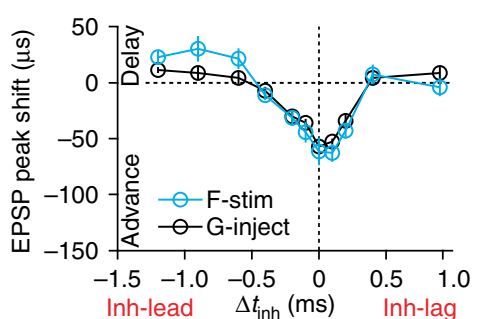

Figure 1 | Inhibition modulates the timing of excitation. (a) Fluorescence micrograph of a P60 MSO neuron. Scale bar, $50 \mu \mathrm{m}$.

(b) Schematic of input circuitry to the MSO. Ipsilateral (Ipsi) and contralateral (Contra) glutamatergic inputs from the anteroventral cochlear nuclei (AVCN) target the dendrites. Glycinergic inputs from the lateral and medial nucleus of the trapezoid body (LNTB and MNTB, respectively) target the soma. (c,d) Schematics of configurations for synaptic stimulation of EPSPs (F-stim) while simulating IPSPs (G-inject) with conductance-clamp (G-clamp, c) and simulating both EPSPs and IPSPs with conductance-clamp (d). (e) Timing convention. The onset of Inhibition is timed relative to that of Excitation from one side $\left(\Delta t_{\mathrm{inh}}\right)$, designated as contralateral. Negative values indicate Inhibition leads Excitation (Inh-lead), and positive values indicate Inhibition lags Excitation (Inh-lag). (f,g) Voltage traces for an example recording using configurations in $\mathbf{c}, \mathbf{d}$, respectively, aligned in time to the peak of the EPSP alone (light traces). The influence of a precisely timed IPSP (red traces) on EPSP peak timing is visualized as a shifted peak of the composite PSP (dark traces). Traces are separated to illustrate inhibitory timing conditions that enforced a peak advance (left) or delay (right). Traces are also plotted aligned in time to the IPSP in Supplementary Fig. 2e. Resting membrane potential $\left(V_{\text {rest }}\right):-64 \mathrm{mV}$. (h) Average ( \pm s.e.m.) EPSP peak shifts plotted against $\Delta t_{\text {inh }}$ for synaptically evoked (blue) and conductance-clamp-simulated (black) EPSPs. Negative and positive values indicate an EPSP peak advance and delay, respectively. $\Delta t_{\text {inh }}=0.1 \mathrm{~ms}, P=0.986 ; \Delta t_{\text {inh }}=-0.6 \mathrm{~ms}, P=0.647$; two-way ANOVA, $n=7$ recordings.

Using this advantage, we investigated a broad parameter space in each neuron to gain insight into the mechanism by which the relative timing of inhibition modulates the peak timing of excitation. For example, this allowed us to test whether the wide range of excitatory and inhibitory input kinetics we observed (Supplementary Fig. 1) is relevant for the efficacy of inhibitionenforced EPSP peak shifts (Fig. 2a-f). Here, we compared representative fast (Fig. 2a,b) and slow (Fig. 2c,d) EPSG and IPSG templates with their average-speed $\left(\tau_{\mathrm{EPSG}}=0.3 \mathrm{~ms}, \tau_{\mathrm{IPSG}}=1.5\right.$ $\mathrm{ms}$ ) counterparts (Supplementary Fig. 2), maintaining an E-I ratio of 1:1. Peak shifts were substantially dependent on excitatory input kinetics, where slower excitation $\left(\tau_{\mathrm{EPSG}}=0.5 \mathrm{~ms}\right)$ was comparably more sensitive to peak shifts in both directions $\left(\Delta t_{\text {inh }}=0.1 \mathrm{~ms}:-92 \pm 12\right.$ versus $-58 \pm 6 \mu \mathrm{s}, P=0.003 ; \Delta t_{\text {inh }}=$ $-0.6 \mathrm{~ms}: 32 \pm 8$ versus $2 \pm 6 \mu \mathrm{s}, P=0.011$, two-way ANOVA, $n=5$; Fig. 2a,c,e). Peak shifts were also sensitive to inhibitory input kinetics, where faster inhibition $\left(\tau_{\mathrm{IPSG}}=1.0 \mathrm{~ms}\right)$ was more effective at enforcing the peak delay $\left(\Delta t_{\text {inh }}=-0.6 \mathrm{~ms}: 20 \pm 6\right.$, $P=0.015$, two-way ANOVA, $n=5)$, but did not substantially impact the peak advance $\left(\Delta t_{\text {inh }}=0.1 \mathrm{~ms}:-62 \pm 8, P=0.925\right.$, two-way ANOVA, $n=5$; Fig. 2b,d,f). Thus, although we predominantly use the average-speed EPSG and IPSG throughout the manuscript, slower excitation and faster inhibition will generally enhance the impact of inhibition on excitatory timing.

Because inhibition can impose both a shunt and hyperpolarization on the membrane, we next evaluated their independent contributions to EPSP peak shifts (Fig. 2g). Shunting inhibition was isolated by hyperpolarizing neurons to the set reversal potential for chloride $(-90 \mathrm{mV})^{20}$. This manipulation revealed a peak advance regardless of timing $\left(\Delta t_{\mathrm{inh}}=0.1 \mathrm{~ms}:-32 \pm 4 \mu \mathrm{s}\right.$; $\Delta t_{\text {inh }}=-0.6 \mathrm{~ms}:-23 \pm 4 \mu \mathrm{s}, n=11$; Fig. $2 \mathrm{~g}$, dotted black). Depolarizing neurons from this point revealed the contribution of hyperpolarization that manifested itself as a biphasic function superimposed on the shunt-induced function. Compared with the median resting potential $(-65 \mathrm{mV}$, Fig. $2 \mathrm{~g}$, maroon and Supplementary Fig. 4) more depolarized potentials $(-45 \mathrm{mV}$, black) revealed larger peak shifts in both directions $\left(\Delta t_{\text {inh }}=0.1 \mathrm{~ms}: \quad-101 \pm 3 \quad\right.$ versus $\quad-67 \pm 4 \mu \mathrm{s}, \quad P<0.001$; $\Delta t_{\text {inh }}=-0.6 \mathrm{~ms}: 17 \pm 6$ versus $-10 \pm 5 \mu \mathrm{s}, P<0.001$, two-way ANOVA, $n=11$; Fig. $2 \mathrm{~g}$ ). We also doubled the E-I ratio to reduce the relative strength of inhibition and found that peak shifts were proportionally reduced, particularly for the peak advance (at $-65 \mathrm{mV}, \Delta t_{\mathrm{inh}}=0.1 \mathrm{~ms}:-42 \pm 4 \mu \mathrm{s}, \quad P=0.002$; $\Delta t_{\text {inh }}=-0.6 \mathrm{~ms}: \quad-3 \pm 6 \mu \mathrm{s}, \quad P=0.972$, two-way ANOVA, $n=11$; Fig. 2h). Thus, an inhibitory shunt generally advances the peak of an EPSP, but hyperpolarization enforces the bidirectional effect of inhibition on EPSP peak timing, depending on the balance of excitation and inhibition. Here, more depolarized membrane potentials (imposing a larger driving force on IPSPs) and stronger inhibition (relative to excitation) enhance the inhibitory control of excitatory timing.

Because MSO neurons integrate their synaptic inputs linearly ${ }^{8,26}$, these findings can be explained by a simple model (Fig. 2i,j). Here, we schematized an EPSP as a Gaussian function 
and the hyperpolarizing profile of an IPSP as a sloped line (in this case the re-depolarizing phase). If we add the line to the Gaussian function, the peak of the summated function is shifted proportionally towards the slope of the line (Fig. 2i). This explains why coincident or slightly lagging inhibition (imposing a negative slope) enforces a peak advance, and why leading inhibition (positive slope) enforces a peak delay. It also illustrates why faster and stronger inhibition (steeper slope) is
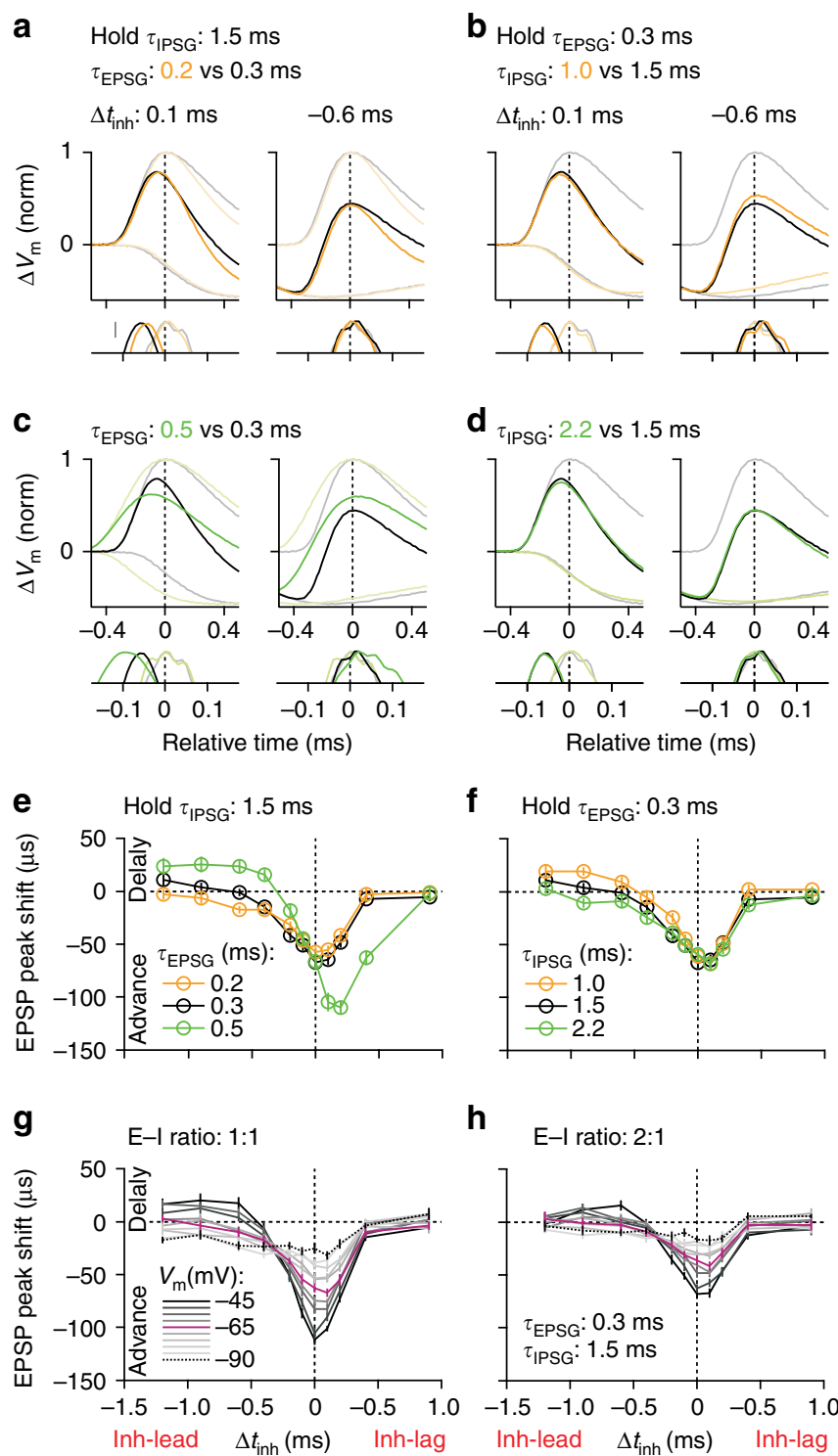

h
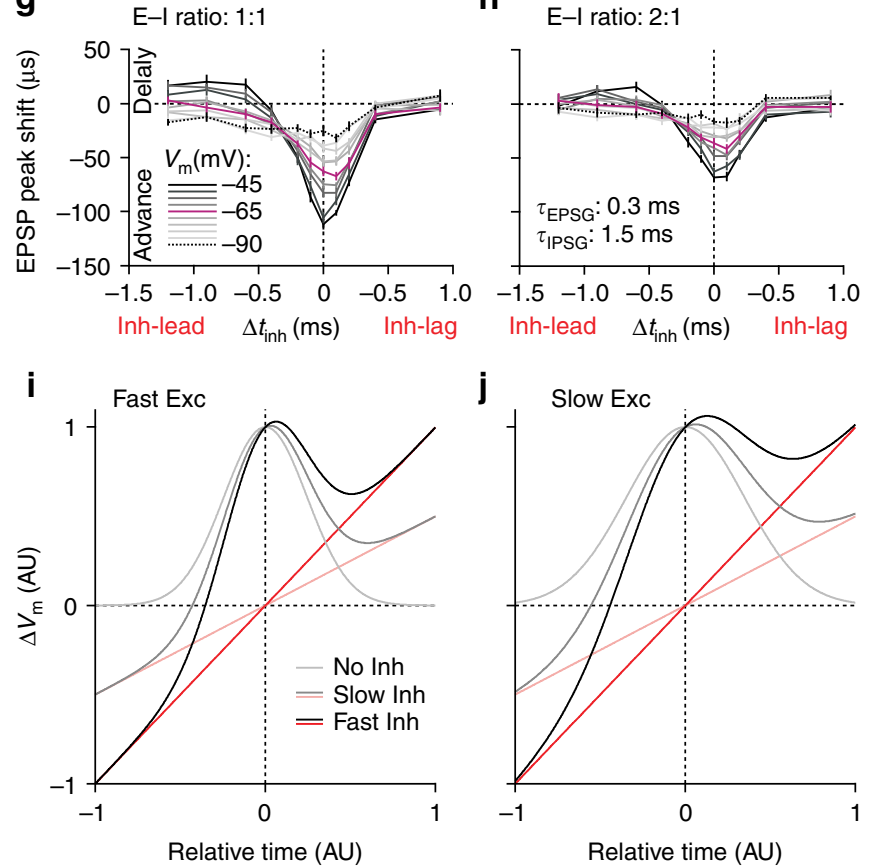

more effective at enforcing EPSP peak shifts ${ }^{22}$. Because differences in inhibitory kinetics predominantly influence the slope of the re-depolarizing phase of the IPSP (Supplementary Fig. 2), it further shows why inhibitory kinetics predominantly influenced the peak delay compared to the peak advance. Finally, if we double the Gaussian half-width to mimic a relatively slower EPSP, larger shifts are generated (Fig. 2j). This explains why slower excitation is more sensitive to peak shifts. Thus, inhibitory control of excitatory timing is influenced by factors beyond the relative time of arrival $6,7,22,28$.

The activity of potassium channels facilitates peak shifts. Before investigating the consequences of inhibition-enforced EPSP peak shifts on bilateral coincidence detection, we investigated the role of low-threshold potassium channels $\left(\mathrm{K}_{\mathrm{LTA}}\right)$ in inhibitionenforced EPSP peak shifts. $\mathrm{K}_{\mathrm{LTA}}$ has been implicated in sharpening EPSPs ${ }^{32,38}$ and has recently been shown to interact with IPSPs ${ }^{26}$. Simply blocking $\mathrm{K}_{\mathrm{LTA}}$ does not allow an assessment of its influence on synaptic integration because $\mathrm{K}_{\mathrm{LTA}}$ also maintains the neuron's exquisitely fast membrane time constant in the adult $(\sim 180 \mu \mathrm{s})$. To circumvent this pharmacological limitation, we generated a point-neuron model based on our electrophysiological measurements and performed cell-wise fittings of EPSPs and IPSPs that were recorded in conductanceclamp (Fig. 3). The model recapitulated the kinetic profile and amplitude of measured events (Fig. 3a) by altering only a few independent parameters (Fig. 3b,c), demonstrating its accuracy for evaluating synaptic integration. It also successfully recapitulated inhibition-enforced EPSP peak shifts for individual recordings (Fig. 3d) and the entire population (Fig. 3e).

To determine whether native $\mathrm{K}_{\mathrm{LTA}}$ activity influenced inhibition-enforced EPSP peak shifts, we eliminated the $K_{\mathrm{LTA}}$

Figure 2 | Input speed and balance influence peak shift magnitude. (a-d) Normalized (norm) voltage traces for an example recording of inhibition-enforced EPSP peak shifts comparing fast $\left(\tau_{\text {EPSG }}=0.2 \mathrm{~ms}\right.$, a; $\tau_{\text {IPSG }}=1.0 \mathrm{~ms}$, b; goldenrod $)$ and slow $\left(\tau_{\text {EPSG }}=0.5 \mathrm{~ms}, \mathbf{c} ; \tau_{\text {IPSG }}=2.2 \mathrm{~ms}\right.$, d; chartreuse) speed EPSGs (a,c) and IPSGs (b,d) with their average-speed counterparts $\left(\tau_{\text {EPSG }}=0.3 \mathrm{~ms} ; \tau_{\text {IPSG }}=1.5 \mathrm{~ms}\right)$ for $\Delta t_{\text {inh }}=0.1$ (left) and $-0.6 \mathrm{~ms}$ (right). Insets are zooms of the peaks, aligned in amplitude. Inset scale bar (a, bottom), $0.1 \mathrm{mV}$. Traces are colour-coded as follows: light traces indicate the EPSP and IPSP alone, dark traces indicate the composite PSP. Traces are aligned in time to the peak of the EPSP alone for each kinetic template pair. $V_{\text {rest }}:-62 \mathrm{mV}$. (e,f) Average ( \pm s.e.m.) EPSP peak shifts plotted against $\Delta t_{\text {inh }}$ for comparing EPSG $\left(\Delta t_{\text {inh }}=0.1 \mathrm{~ms}: \tau_{\text {EPSG }}=0.2 \mathrm{~ms}\right.$, $P=0.542 ; \tau_{\mathrm{EPSG}}=0.5 \mathrm{~ms}, P=0.003 ; \Delta \mathrm{t}_{\mathrm{inh}}=-0.6 \mathrm{~ms}: \tau_{\mathrm{EPSG}}=0.2 \mathrm{~ms}$, $\left.P=0.422 ; \tau_{\mathrm{EPSG}}=0.5 \mathrm{~ms}, P=0.011\right)(\mathbf{e}) ;$ and IPSG $\left(\Delta t_{\mathrm{inh}}=0.1 \mathrm{~ms}\right.$ : $\tau_{\mathrm{IPSG}}=1.0 \mathrm{~ms}, P=0.925 ; \tau_{\mathrm{IPSG}}=2.2 \mathrm{~ms}, P=0.213 ; \Delta t_{\text {inh }}=-0.6 \mathrm{~ms}$ : $\tau_{\text {IPSG }}=1.0 \mathrm{~ms}, P=0.015 ; \tau_{\mathrm{IPSG}}=2.2 \mathrm{~ms}, P=0.500$ ) (f) kinetics. Two-way ANOVA, $n=5$ recordings. (g,h) Same as in e,f, except the average-speed EPSG and IPSG were used, and the holding potential $\left(V_{m}\right)$ was between -90 and $-45 \mathrm{mV}$ in $5 \mathrm{mV}$ increments. Compared with the median $V_{\text {rest }}:-65 \mathrm{mV}$ (maroon; Supplementary Fig. $4 \mathrm{~b}$ ), $V_{m}=-45 \mathrm{mV}$ (black): $\Delta t_{\text {inh }}=0.1 \mathrm{~ms}, P<0.001 ; \Delta t_{\text {inh }}=-0.6 \mathrm{~ms}, P<0.001$. The E-I ratio was 1:1 in e, but 2:1 in f. Compared with E-I ratio $=1: 1, E-I$ ratio $=2: 1$ at $V_{\mathrm{m}}=-65 \mathrm{mV}: \Delta \mathrm{t}_{\mathrm{inh}}=0.1 \mathrm{~ms}, P<0.001 ; \Delta t_{\text {inh }}=-0.6 \mathrm{~ms}, P=0.972$. Two-way ANOVA, $n=11$ recordings. $(\mathbf{i}, \mathbf{j})$ Model illustrating the mechanism underlying the dynamics of Inh-enforced EPSP peak shifts. Fast and slow EPSPs are modelled as Gaussian functions of comparably narrow (i) and wide (j) half-widths, respectively. Slow, weak (pink) and fast, strong (red) IPSPs are modelled as shallow and steep sloped linear functions, respectively. The EPSP alone is shown in light grey with a peak at $t=0$. The composite PSP is the linear sum of the Gaussian function and the shallow (grey) or steep (black) linear functions. AU, arbitrary units. 
activation kinetics, thereby rendering the channel passive. Interestingly, this manipulation did not substantially reduce peak shifts $\left(\Delta t_{\text {inh }}=0.1 \mathrm{~ms}:-66\right.$ versus $-73 \mu \mathrm{s} ; \Delta t_{\text {inh }}=-0.6 \mathrm{~ms}:-6$ versus $-6 \mu \mathrm{s}$; Fig. 3f, turquoise). However, optimal model fit parameters (Fig. 3b) supported by measured intrinsic membrane properties (Supplementary Fig. 4) indicate that in contrast to observations in juvenile (P16-19) gerbils $^{38} \mathrm{~K}_{\mathrm{LTA}}$ channels in adult (P60-P90) gerbils are activated at more hyperpolarized potentials $\left(V_{0.5}=-73 \mathrm{mV}\right)$, thus rendering a larger fraction of them open at rest $(-65 \mathrm{mV})$. Indeed, we found that in contrast to P17 gerbils, the slowing and tightening of the membrane with increasing hyperpolarization (indicating that $\mathrm{K}_{\mathrm{LTA}}$ had closed) was not complete, even at $-90 \mathrm{mV}$ (Supplementary Fig. 4d,e). Interestingly, depolarizing the activation voltage in the model to levels observed in juvenile gerbils $\left(V_{0.5}=-38 \mathrm{mV}\right)^{38}$ revealed more robust peak shifts, particularly for a peak delay

a
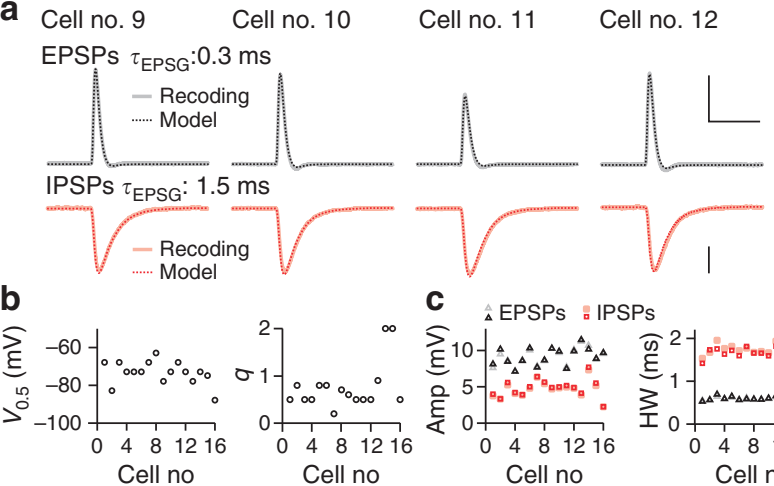

C $A$ EPSPS :IPSPS
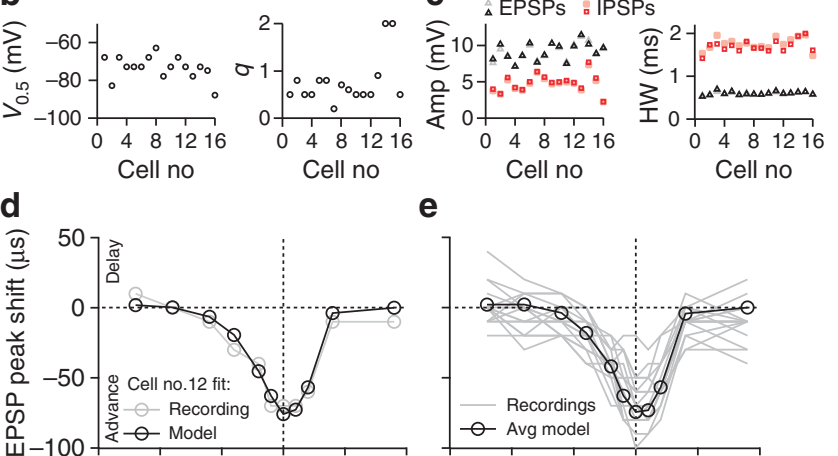

e
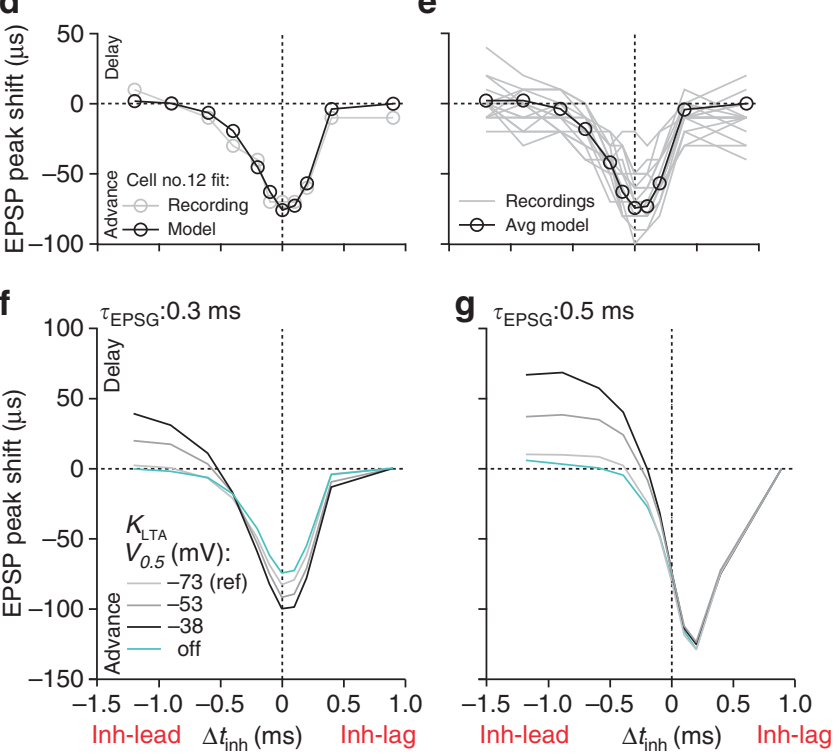

h

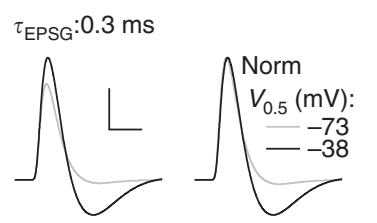

i $\tau_{\text {EPSG }}: 0.5 \mathrm{~ms}$

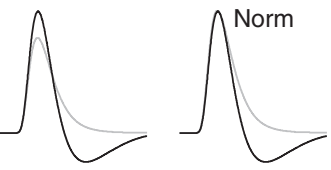

j $\tau_{\text {EPSG }}: 1.5 \mathrm{~ms}$

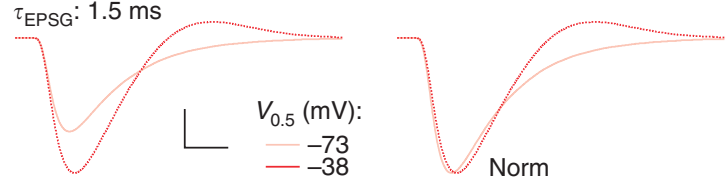

$\left(\Delta t_{\text {inh }}=0.1 \mathrm{~ms}:-90 \mu \mathrm{s} ; \Delta t_{\text {inh }}=-0.6 \mathrm{~ms}: 10 \mu \mathrm{s}\right.$; Fig. 3f, black $)$. We also repeated the same protocol using the slow-speed EPSG and found even more pronounced peak delays $\left(\Delta t_{\mathrm{inh}}=-0.6 \mathrm{~ms}\right.$ : 55 versus $8 \mu$ s; Fig. $3 g$ ).

To gain insight into the mechanism underlying the facilitation of $\mathrm{K}_{\mathrm{LTA}}$ activity on inhibition-enforced EPSP peak shifts, we further evaluated the EPSPs (Fig. 3h,i) and the IPSP (Fig. 3j) produced by the model. When the $\mathrm{K}_{\mathrm{LTA}}$ activation voltage was depolarized, both EPSPs and the IPSP amplitudes increased, but only IPSP kinetics were visibly altered. $\mathrm{K}_{\mathrm{LTA}}$ gating is fast enough to follow the profile of the IPSP and is thus closed during the hyperpolarizing phase. This naturally moves the membrane potential away from the potassium reversal potential, speeding the re-depolarizing phase, from which our previous experiments would predict larger peak delays (Fig. 2b,f,i) ${ }^{39}$. The observation that the slow-speed EPSG is even more sensitive to $\mathrm{K}_{\mathrm{LTA}}$ activity is also consistent with our observation that slower EPSPs are generally more sensitive to peak shifts (Fig. 2c,e,j). Thus, $\mathrm{K}_{\mathrm{LTA}}$ activity promotes inhibition-enforced EPSP peak shifts by speeding the decay kinetics of the IPSP, and therefore may have a more influential role in facilitating E-I interactions during development ${ }^{14,32,38}$.

Inhibition tunes coincidence detection. We have shown that inhibition can modulate the peak timing of excitation from one side, but it was not known how this influences the integration of bilateral excitation. We therefore evaluated the effect of a precisely timed IPSP on subthreshold PSP summation and suprathreshold action potential probability functions (Fig. 4). Because excitation from both sides have equal strength (Supplementary Fig. 1) and summate linearly at the soma ${ }^{8,26}$, we injected two identical EPSGs at various relative times $\left(\Delta t_{\text {exc }}\right)$ to simulate ITDs in vitro (Fig. $4 \mathrm{a}$, see Methods) ${ }^{25,26}$. We then determined whether a single IPSP (timed relative to the contralateral EPSP) could bias the excitatory timing of best PSP summation (PSP sum) or greatest action potential probability (AP prob). For these experiments, it matters at what $\Delta t_{\mathrm{exc}}$ the largest summation or the most action potentials occur and not when they occur, which was the case for previous experiments (Figs 1-3). In the absence of inhibition, these functions peaked near zero (PSP sum: $3 \pm 2 \mu \mathrm{s}$, $n=5$; Fig. 4 b; AP prob: $7 \pm 3 \mu$ s, $n=7$; Fig. $4 \mathrm{c}$ ).

Contralateral inhibition modulated coincidence detection timing in a manner consistent with its effects on the peak timing

Figure 3 | The activity of potassium channels facilitates peak shifts. (a) Example model fittings of EPSPs (top) and IPSPs (bottom) overlaid with corresponding experimentally recorded responses (dotted lines). Scale bars, $5 \mathrm{mV}, 5 \mathrm{~ms}$ (top) and $2 \mathrm{mV}$ (bottom). $V_{\text {rest }}:-65 \mathrm{mV}$. (b) Halfactivation voltage $\left(V_{0.5}\right.$, left $)$ and the multiplicative scaling factor for the activation time constant ( $q$, right) of the $\mathrm{K}_{\mathrm{LTA}}$ values for each cell in the population ( $n=16$ cells). (c) Comparisons between model-fitted (dark markers) and recorded (light markers) EPSP and IPSP amplitude (Amp, left) and half-width (HW, right). (d) EPSP peak shifts plotted against $\Delta t_{\text {inh }}$ for the model prediction (black) and experimental values (grey) for an example cell fitting. (e) Same as in $\mathbf{d}$, but the prototypical neuron generated from average fit parameters (black) compared with the population of recordings (grey). (f) Same as in $\mathbf{d}$, but the $\mathrm{K}_{\mathrm{LTA}} V_{0.5}$ was depolarized from the reference value for the example cell in $\mathbf{d}(-73 \mathrm{mV}$, light grey) to -53 (grey) and -38 (black) $m V$. The activation kinetics of the $K_{\text {LTA }}$ was also slowed by 100-fold to eliminate $K_{\text {LTA }}$ activity (turquoise). (g) Same as in $\mathbf{f}$, but for the slow-speed EPSG. $(\mathbf{h}-\mathbf{j})$ Voltage traces of the EPSPs $(\mathbf{h}, \mathbf{i})$ and IPSP $(\mathbf{j})$ produced by the model of the cell in $(\mathbf{d}, \mathbf{f}, \mathbf{g})$ with a $\mathrm{K}_{\text {LTA }} V_{0.5}$ of -73 (light traces) or $-38 \mathrm{mV}$ (dark traces, left). Normalized (Norm) traces are shown on the right to illustrate differences in kinetics. Scale bars, $5 \mathrm{mV}, 1 \mathrm{~ms}$ $(\mathbf{h}, \mathbf{i})$, and $2 \mathrm{mV}, 1 \mathrm{~ms}(\mathbf{j})$. 


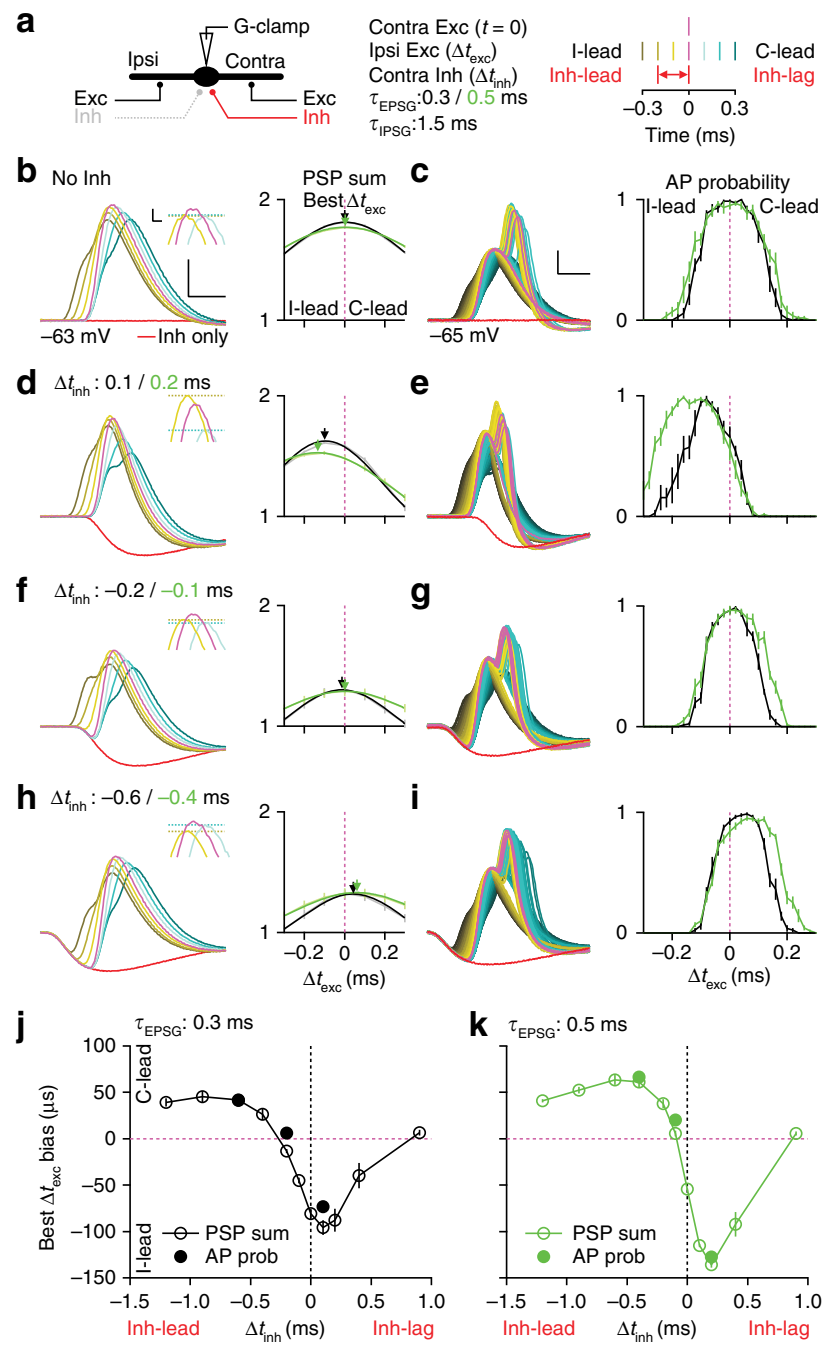

Figure 4 | Inhibition tunes coincidence detection. (a) Schematic illustrating three of four inputs examined (left) and timing convention (right). Contralateral Inh and ipsilateral excitation are timed relative to Contra Exc ( $\Delta t_{\text {inh }}$ and $\Delta t_{\text {exc }}$ respectively). (b,d,f,h) Voltage traces for an example recording of a subthreshold PSP summation experiment (left). Traces were generated from the average-speed EPSG and IPSG and are colour-coded to reflect excitatory timing conditions in a. Scale bar, $5 \mathrm{mV}$, $1 \mathrm{~ms}$. Insets show zooms of the peaks. Summation bias is resolved by comparing the peak amplitudes of the flanking $100 \mu \mathrm{s}$ Ipsilateral-leading (yellow) and contralateral-leading (cyan) traces (horizontal dotted lines) against zero $\Delta t_{\text {exc }}$ (pink). Inset scale bar, $50 \mu \mathrm{V}, 0.1 \mathrm{~ms}$. Summated PSP amplitudes (PSP sum, normalized to the response of a single EPSP) are plotted against $\Delta t_{\text {exc }}$ (light) and fitted with Gaussian functions (dark) for experiments using the average-(j) and slow (k)-speed EPSG (right). Arrows indicate the peak of the function fits (best $\Delta t_{\text {exc }}$ ). Negative and positive $\Delta t_{\text {exc }}$ values indicate Ipsi-leading (I-lead) and Contra-leading (C-lead) conditions, respectively. Contra Inh was absent from (b) or occurred at $\Delta t_{\text {inh }}$ values for average and slow-speed EPSGs that (respectively) advanced $(0.1 / 0.2 \mathrm{~ms}$, d), did not substantially shift $(-0.2 /-0.1 \mathrm{~ms}, \mathbf{f})$ or delayed $(-0.6 /-0.4 \mathrm{~ms}, \mathbf{h})$ the peak of the Contra EPSP. Different $\Delta t_{\text {inh }}$ values are shown for the average and slow EPSGs because these representative effects occurred at different relative timing conditions. $n=5$ recordings. (c,e,g,i) Same as in $\mathbf{b}, \mathbf{d}, \mathbf{f}, \mathbf{h}$, except that suprathreshold action potential probability (AP prob) functions were determined. Scale bar, $5 \mathrm{mV}, 1 \mathrm{~ms} ; n=8-12$ recordings. (j,k) Best $\Delta t_{\text {exc }}$ ( \pm s.d.) plotted against $\Delta t_{\text {inh }}$ for average-(j) and slow (k)-speed EPSGs for PSP summation experiments (open markers) and action potential probability experiments (filled markers). of contralateral excitation. Inhibitory timing conditions that advanced the peak of the contralateral EPSP $\left(\Delta t_{\text {inh }}=0.1 \mathrm{~ms}\right)$ biased subthreshold summation and action potential probability functions towards ipsilateral-leading excitation (PSP sum: $-96 \pm 8 \mu \mathrm{s}, \quad n=5$; Fig. 4d; AP prob: $-73 \pm 2 \mu \mathrm{s}, n=7$; Fig. 4e). This corresponds to an advanced contralateral EPSP that now summates best with an ipsilateral EPSP that occurs earlier in time. Timing conditions that did not robustly influence the peak timing of the EPSP $\left(\Delta \mathrm{t}_{\mathrm{inh}}=-0.2 \mathrm{~ms}\right)$ did not substantially bias coincidence detection (PSP sum: $-11 \pm 2 \mu \mathrm{s}$, $n=5$; Fig. 4f; AP prob: $6 \pm 3 \mu \mathrm{s}, n=8$; Fig. $4 \mathrm{~g}$ ), consistent with a different study that was performed with synaptic stimulation ${ }^{26}$. However, this timing condition $\left(\Delta t_{\mathrm{inh}}=-0.2 \mathrm{~ms}\right)$ did narrow the half-widths of the coincidence detection functions (PSP sum: $387 \pm 7$ versus $467 \pm 6 \mu \mathrm{s}, P<0.001, n=5$; AP prob: $208 \pm 6$ versus $221 \pm 8 \mu \mathrm{s}, P<0.001$, two-way ANOVA, $n=8$ ), also consistent with the synaptic stimulation experiments ${ }^{26}$. Finally, timing conditions that delayed the peak of the contralateral EPSP $\left(\Delta t_{\text {inh }}=-0.6 \mathrm{~ms}\right)$ biased coincidence detection towards contralateral-leading excitation, albeit to a lower extent (PSP sum: $41 \pm 6 \mu \mathrm{s}, n=5$; Fig. $4 \mathrm{~h}$; AP prob: $41 \pm 2 \mu \mathrm{s}, n=8$; Fig. $4 \mathrm{i})$. This corresponds to a delayed contralateral EPSP that now summates best with an ipsilateral EPSP that occurs later in time. Thus, a precisely timed contralateral IPSP can modulate coincidence detection in a bidirectional manner, but only under timing conditions that had not been previously explored ${ }^{26}$.

Because recent in vivo recordings of MSO neurons revealed many cases where the EPSP was much slower than our average in vitro measurements ${ }^{8}$, we also evaluated the slow-speed EPSG paired with the average-speed IPSG (Fig. $4 \mathrm{~b}-\mathrm{i}, \mathrm{k}$, chartreuse). The peaks of both PSP summation and action potential probability functions were biased comparably further towards ipsilateralleading $\left(\Delta t_{\mathrm{inh}}=0.2 \mathrm{~ms}\right.$, PSP sum: $-136 \pm 2 \mu \mathrm{s}, n=5$; AP prob: $-127 \pm 3 \mu \mathrm{s}, n=7)$ and contralateral-leading $\left(\Delta t_{\mathrm{inh}}=-0.4 \mathrm{~ms}\right.$, PSP sum: $64 \pm 5 \mu \mathrm{s}, n=5$; AP prob: $66 \pm 3 \mu \mathrm{s}, n=12$ ) excitation. This is consistent with the observation that slower EPSPs are more sensitive to inhibition-enforced peak shifts (Fig. 2c,e). Importantly, the similarity between the peaks of subthreshold and suprathreshold coincidence detection functions (Fig. 4j,k) indicates that the influence of inhibition on subthreshold summation accurately translates into action potential generation.

Synaptic jitter enhances peak shifts. Because MSO neurons can be ITD-sensitive at frequencies between $\sim 100$ and $\sim 1,500 \mathrm{~Hz}$ (ref. 48) we returned to our single-side E-I interaction paradigm (Figs 1-3) and evaluated the efficacy of inhibitory modulation of excitatory timing under such naturalistic conditions. Although phase locking is relatively stronger at low frequencies, synaptic inputs are actually more jittered ${ }^{39}$. We therefore challenged our inhibition-enforced EPSP peak shift paradigm with increasing amounts of jitter (Fig. 5). We introduced jitter to four EPSGs and IPSGs with gamma distribution functions of up to $640 \mu \mathrm{s}$ in halfwidth (Fig. 5a,b). The resulting composite EPSPs and IPSPs revealed a broadening of event kinetics with increasing jitter, but much more pronounced for EPSPs than IPSPs (Fig. 5c). Increasing excitatory jitter alone robustly increased peak shifts in both directions (for $320 \mu \mathrm{s}$ excitatory jitter, $\Delta t_{\text {inh }}=0.1 \mathrm{~ms}$ : $117 \pm 8$ versus $67 \pm 6 \mu \mathrm{s}, \quad P<0.001 ; \quad \Delta \mathrm{t}_{\text {inh }}=-0.6 \mathrm{~ms}: 44 \pm 4$ versus $6 \pm 3 \mu \mathrm{s}, P=0.002$, two-way ANOVA, $n=6$; Fig. $5 \mathrm{~d}$,e, blue), and increasing inhibitory jitter alone decreased peak shifts (for $320 \mu \mathrm{s}$ inhibitory jitter, $\Delta t_{\text {inh }}=0.1 \mathrm{~ms}$ : $-48 \pm 4 \mu \mathrm{s}, P=0.023$; $\Delta t_{\text {inh }}=-0.6 \mathrm{~ms}:-5 \pm 3 \mu \mathrm{s}, P=0.344$, two-way ANOVA, $n=6$; Fig. 5d,e, red). Interestingly, increasing both excitatory and inhibitory jitter resulted in a net increase in peak shifts (for $320 \mu \mathrm{s}$ excitatory plus inhibitory jitter, $\Delta t_{\text {inh }}=0.1 \mathrm{~ms}:-95 \pm 9 \mu \mathrm{s}$, 
a

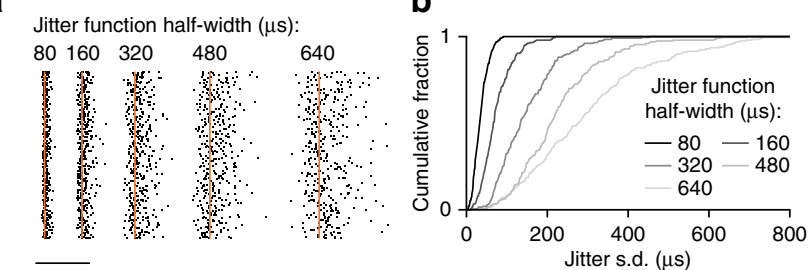

C

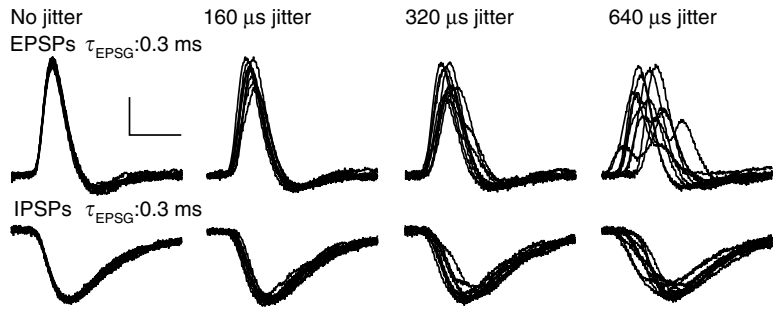

d No jitter
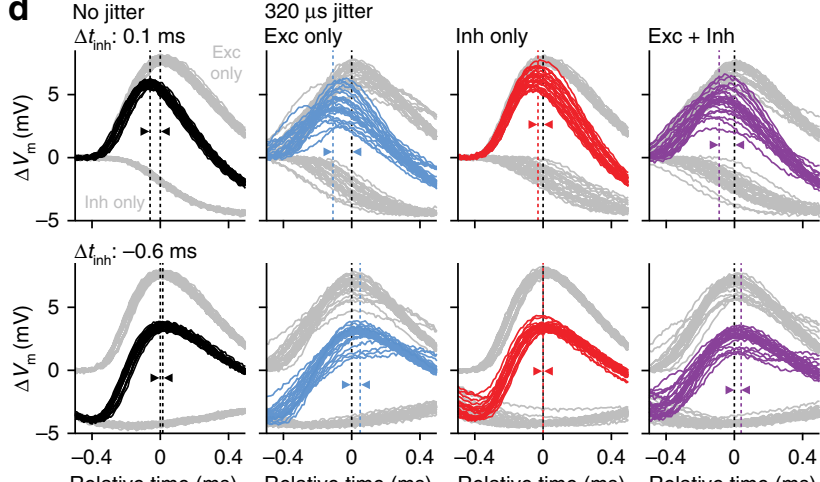

Relative time (ms) Relative time (ms) Relative time (ms) Relative time (ms)

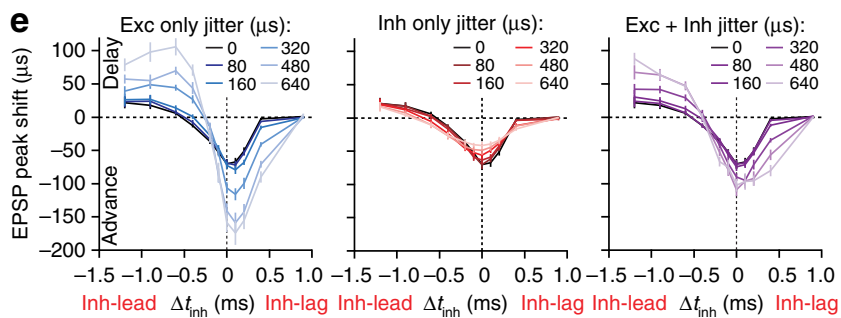

Figure 5 | Synaptic jitter enhances peak shifts. (a) Raster plots showing 100 trials of four event onset times jittered with gamma distributions of increasing function half-widths (left to right). Orange line indicates the reference time point (no jitter). Scale bar, $1 \mathrm{~ms}$. (b) Cumulative histogram of the onset timing s.d. for the four jittered events. (c) Ten overlaid voltage traces for an example recording of the resulting composite EPSPs (top) and IPSPs (bottom) generated by jitter functions of increasing half-widths (left to right). $V_{\text {rest }}:-64 \mathrm{mV}$. Scale bar, $2 \mathrm{mV}, 1 \mathrm{~ms}$. (d) Voltage traces of 25 trials from the recording in c (from left to right) without jitter, or the $320 \mu \mathrm{s}$ half-width jitter function applied to excitation (Exc) only, inhibition (Inh) only, or Exc and Inh. Inhibitory timing conditions that advanced $\left(\Delta t_{\text {inh }}=0.1 \mathrm{~ms}\right.$, top) and delayed ( $\Delta t_{\text {inh }}=-0.6 \mathrm{~ms}$, bottom) EPSP peak timing are shown. Grey traces show EPSPs and IPSPs alone, and dark traces show composite PSPs, aligned in time to the peak of the EPSP alone for each trial. Dotted vertical lines and arrow heads indicate the average peak shift of all 100 trials in the recording. (e) Average ( \pm s.e.m.) EPSP peak shifts plotted against $\Delta t_{\text {inh }}$ for each jitter function applied to Exc only (left), Inh only (centre) and Exc plus Inh (right). Without jitter, EPSP peak shifts were similar to those in Fig. 1h. Compared with no jitter conditions, introducing EPSP jitter ( $320 \mu \mathrm{s}$ jitter function half-width) increased peak shifts to $\sim 200 \%\left(\Delta t_{\text {inh }}=0.1 \mathrm{~ms}, P<0.001 ; \Delta t_{\text {inh }}=-0.6 \mathrm{~ms}, P=0.002\right)$, and introducing IPSP jitter reduced peak shifts to $\sim 75 \%\left(\Delta t_{\text {inh }}=0.1 \mathrm{~ms}\right.$, $\left.P=0.023 ; \Delta t_{\text {inh }}=-0.6 \mathrm{~ms}, P=0.344\right)$. Introducing EPSP plus IPSP jitter increased peak shifts to $\sim 150 \%\left(\Delta t_{\text {inh }}=0.1 \mathrm{~ms}, P=0.014\right.$; $\left.\Delta \mathrm{t}_{\text {inh }}=-0.6 \mathrm{~ms}, P=0.047\right)$. Two-way ANOVA, $n=6$ recordings.
$P=0.014 ; \quad \Delta t_{\text {inh }}=-0.6 \mathrm{~ms}: \quad 44 \pm 4 \mu \mathrm{s}, \quad P=0.047, \quad$ two-way ANOVA, $n=6$; Fig. 5d,e, magenta). Evaluation of individual trials revealed that substantially jittered conditions increase the diversity of peak shift magnitudes (Supplementary Fig. 5). However, the distribution of all trials (Supplementary Fig. 6) shows a trend towards larger peak shifts with equal amounts of excitatory and inhibitory jitter. The ability of excitatory jitter to outcompete the reduction in peak shifts generated by inhibitory jitter can be explained by larger distortion of the EPSP profile compared to the IPSP profile with equivalent amounts of jitter (Fig. 5c), from which our analysis of synaptic kinetics (Fig. 2) predict larger peak shifts. These findings indicate that naturally occurring synaptic jitter enhances inhibition-enforced EPSP peak shifts, despite the degradation of peak timing precision that was recently suggested to diminish its efficacy ${ }^{8,26}$. As ITD tuning at low frequencies has been particularly difficult to explain by axon length disparity ${ }^{11}$, these findings support an especially important role of inhibition for ITD tuning in the low-frequency range ${ }^{40}$.

Precise inhibition maintains efficacy at high frequencies. At high frequencies, synaptic jitter is much less, but IPSPs naturally summate ${ }^{26}$. We thus challenged inhibition-enforced EPSP peak shifts with 16 pulse trains at 333, 500 and $800 \mathrm{~Hz}$ under timing conditions that produced a large peak advance $\left(\Delta t_{\text {inh }}=0.1 \mathrm{~ms}\right)$ and delay $\left(\Delta t_{\text {inh }}=-0.6 \mathrm{~ms}\right) \quad$ (Fig. 6). Despite substantial summation (Fig. 6a, red, and Supplementary Fig. 7), inhibition sustained the ability to advance (Fig. 6b, left) and delay (Fig. 6b, right) EPSP peak timing, even at $800 \mathrm{~Hz}$ (for the 16th event, $\Delta t_{\text {inh }}=0.1 \mathrm{~ms}:-48 \pm 2 \mu \mathrm{s} ; \Delta t_{\text {inh }}=-0.6 \mathrm{~ms}: 22 \pm 6 \mu \mathrm{s}, \quad n=8$; Fig. $6 \mathrm{~b}$, bottom). Small changes in peak shifts during the train (16th event compared with the first, $\Delta t_{\text {inh }}=0.1 \mathrm{~ms}:-70 \pm 4 \mu \mathrm{s}$, $P<0.001 ; \quad \Delta t_{\text {inh }}=-0.6 \mathrm{~ms}: \quad 18 \pm 4 \mu \mathrm{s}, \quad P=0.996$, two-way ANOVA, $n=8$; Fig. $6 c)$ can be attributed to frequencydependent interactions between individual IPSPs during the train, which alter their effective relative peak timing (Supplementary Fig. 7). Inhibition-enforced subthreshold PSP summation bias was also similarly maintained at $800 \mathrm{~Hz}$ (Supplementary Fig. 8), strengthening the translation from inhibition-enforced EPSP peak shifts to coincidence detection timing. Importantly, at higher frequencies IPSPs cannot be discerned during the train regardless of whether they lead or lag EPSPs (Fig. 6a, bottom and Supplementary Fig. 8a, bottom). This is remarkably consistent with the inability to detect IPSPs from in vivo whole-cell recordings ${ }^{8}$. Thus, inhibition maintains its ability to influence EPSP peak timing and synaptic integration at high frequencies, despite undergoing substantial summation that was recently suggested to diminish its efficacy ${ }^{26}$.

Bilateral inhibition extends the range of tuning. To this point, we have considered only the influence of one (contralateral) inhibitory pathway on synaptic integration and coincidence detection. However, in the full MSO circuit both ipsilateral and contralateral inhibition are present ${ }^{31,41}$. We therefore examined the additional effect of a second, precisely timed (putative ipsilateral) inhibition in biasing subthreshold PSP summation (Fig. 7a). In these experiments, we timed the ipsilateral IPSP relative to its corresponding $\operatorname{EPSP}\left(\Delta t_{\text {inhi }}\right)$ and used the slowspeed EPSG paired with the average-speed IPSG to evaluate the extent of inhibitory modulation on synaptic integration under our experimental conditions (Fig. 7b). Importantly, an ipsilateral IPSP will also interact with the contralateral EPSP, but because it is locked in time to the ipsilateral EPSP, the integration of all four synaptic inputs can be conceptually simplified as two composite PSPs from each side that summate linearly at the soma. 
a $333 \mathrm{~Hz} \tau_{\text {EPSG }}: 0.3 \mathrm{~ms} \tau_{\text {IPSG }}: 1.5 \mathrm{~ms}$
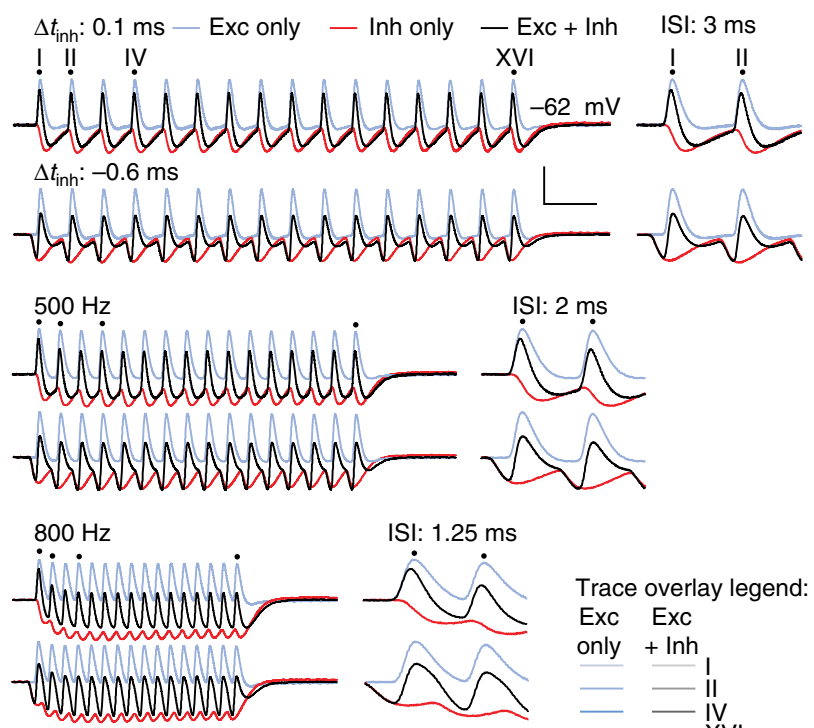

Trace overlay legend:

Exc Exc

only + Inh

- - II

$-\quad-\mathrm{IV}$ b
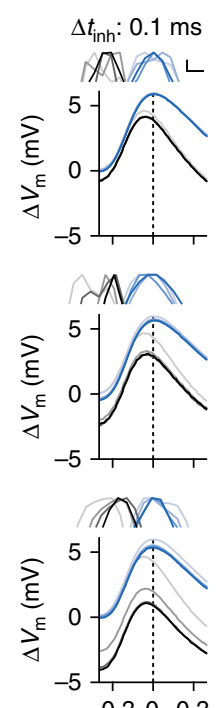

$\begin{array}{lll}0.3 & 0 & 0.3\end{array}$

Relative time (ms)
C
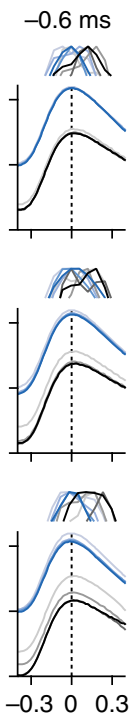

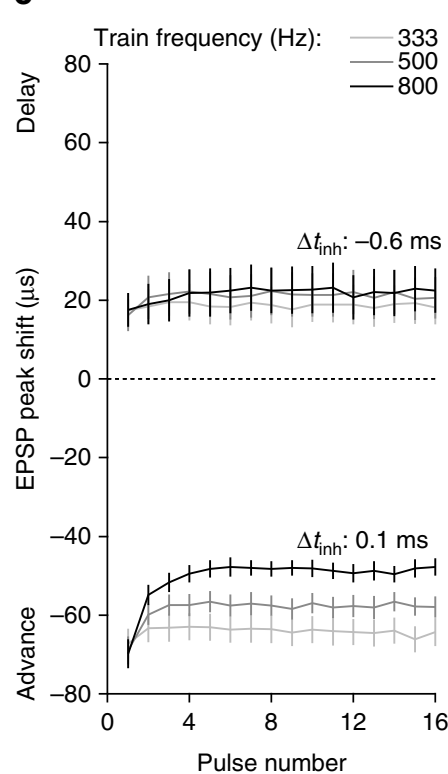

Figure 6 | Precise inhibition maintains efficacy at high frequencies. (a) Voltage traces of 16 pulse trains for an example recording of EPSPs (blue), IPSPs (red) and composite PSPs (black) at 333 (top), 500 (middle) and 800 (bottom) Hz. For each frequency, inhibitory timing that enforced an EPSP peak advance ( $\Delta t_{\mathrm{inh}}=0.1 \mathrm{~ms}$, upper traces $)$ and delay $\left(\Delta t_{\mathrm{inh}}=-0.6 \mathrm{~ms}\right.$, lower traces $)$ are shown. Dots and numerals above the traces indicate individual events that are further analysed in $\mathbf{b}$. Scale bar, $5 \mathrm{mV}, 5 \mathrm{~ms}$. The entire train is shown on the left, and a time zoom of the first two events is shown on the right. ISI, inter-stimulus interval. (b) The first, second, fourth and sixteenth event for each train in a overlaid for the peak advance (left) and delay (right) conditions, aligned in time to the peak of the EPSP alone. Traces are colour-coded to indicate their position in the train (colour code is indicated in a, bottom right). IPSPs are omitted for clarity. Insets above each plot are zooms of the peaks, aligned in amplitude. Inset scale bar, $50 \mu \mathrm{V}, 50 \mu \mathrm{s}$. (c) Average ( \pm s.e.m.) inhibition-enforced EPSP peak shifts plotted against each event in the train for peak advance $\left(\Delta t_{\text {inh }}=0.1 \mathrm{~ms}\right)$ and delay $\left(\Delta t_{\text {inh }}=-0.6 \mathrm{~ms}\right)$ conditions. At $800 \mathrm{~Hz}$, the 16th event compared with the first: $\Delta t_{\text {inh }}=0.1 \mathrm{~ms}, P<0.001 ; \Delta t_{\text {inh }}=-0.6 \mathrm{~ms}, P<0.001$; two-way ANOVA, $n=8$ recordings. Independent analyses of EPSP and IPSP trains are shown in Supplementary Fig. 7. Inh, Inhibition.

We started with a pre-existing contralateral inhibition that was timed (now termed $\Delta t_{\text {inhc }}$ ) to bias ipsilateral-leading excitation $\left(\Delta t_{\text {inhc }}=0.2 \mathrm{~ms}:-163 \pm 2 \mu \mathrm{s}, n=7\right.$; Fig. $\left.7 \mathrm{c}-\mathrm{e}\right)$, produce no bias $\left(\Delta t_{\text {inhc }}=-0.1 \mathrm{~ms}:-2 \pm 4 \mu \mathrm{s}, n=7\right.$; Fig. $\left.7 \mathrm{f}-\mathrm{h}\right)$ or bias contralateral-leading excitation $\left(\Delta t_{\text {inhc }}=-0.4 \mathrm{~ms}: 71 \pm 7 \mu \mathrm{s}\right.$, $n=7$; Fig. $7 \mathrm{i}-\mathrm{k}$ ). In each case, the additional ipsilateral IPSP biased the timing of best PSP summation in a manner nearly equal and opposite to that of the contralateral IPSP at each $\Delta t_{\text {inhc }}$ tested. This is consistent with an ipsilateral IPSP that shifts the peak timing of the ipsilateral EPSP in the same manner as for the contralateral side, which would in fact produce the inverse influence on coincidence detection timing (Fig. 8a). Now considering the temporal integration of all four synaptic inputs, inhibition can bias coincidence detection up to $220 \pm 10 \mu \mathrm{s}$ towards ipsilateral-leading excitation $\left(\Delta t_{\text {inhc }}=0.2\right.$ and $\Delta t_{\text {inhi }}=$ $-0.4 \mathrm{~ms}$; Fig. 7e) and up to $217 \pm 19 \mu$ s towards contralateralleading excitation $\left(\Delta t_{\text {inhc }}=-0.4\right.$ and $\Delta t_{\text {inhi }}=0.2 \mathrm{~ms}$; Fig. $\left.7 \mathrm{k}\right)$. Remarkably, this $>400 \mu$ s dynamic range is achieved within a timing window of just $0.6 \mathrm{~ms}$ (Fig. 8a). This is important, because even though we do not know what other internal delays may be generated in the circuit, inhibition can provide additional timing shifts that largely span the distribution of gerbil ITD functions recorded in vivo (Fig. 8b $)^{6-8,43}$, which has been thus far unsuccessful by axon length disparity-based models of ITD tuning ${ }^{11}$. Interestingly, at timing conditions where inhibition is most capable of influencing coincidence detection, IPSPs would largely be masked by EPSPs, which is compatible with the in vivo observation that even at low frequencies IPSPs remained undetected between cycles ${ }^{8}$. Thus, it is tempting to speculate that the strong afterhyperpolarizations following EPSPs recorded in $v i v o^{8}$ reflect the re-depolarization phase of a precisely timed IPSP that occurs nearly concomitant.

\section{Discussion}

We demonstrated that precise inhibition modulates coincidence detection timing in MSO neurons, supporting a crucial role for inhibition in ITD tuning at the cellular level. The cellular basis for the near microsecond discrimination of ITDs, an essential component of the ability to localize sounds in mammals is elusive. As it has recently become clear that axon length disparity alone cannot predict the preferred ITD of individual MSO neurons ${ }^{11}$, several alternative models were proposed ${ }^{7,14,15}$

The original inhibition-based model was grounded on the finding that blocking glycinergic inhibition in vivo shifted contralateral-leading ITD functions towards $\mathrm{zero}^{6-8}$. It was thought that a leading contralateral inhibition predominantly mediates this effect by delaying the effective peak timing of contralateral excitation ${ }^{6,724,28}$. This mechanism was questioned because it required unusually fast and artificial IPSP kinetics ${ }^{15}$ and a level of temporal precision that would be diminished by synaptic jitter and summation ${ }^{8,26}$. We therefore based our re-evaluation on measured, mature synaptic responses (Fig. 1) under challenging temporal conditions (Figs 5 and 6). We found that inhibition was indeed capable of modulating the timing of excitation, but that a leading contralateral inhibition alone only modestly biased coincidence detection timing towards the contralateral-leading side ( $\sim 50 \mu \mathrm{s}$; Fig. $4 \mathrm{~h}, \mathrm{i})$. However, adding the ipsilateral source of inhibition ${ }^{31,41}$, which has received much less attention, could bias coincidence detection a further $\sim 150 \mu \mathrm{s}$ towards the contralateral-leading side (Fig. 7k). These data strongly support the in vivo pharmacological experiments ${ }^{6,7}$, but demonstrate that the precise timing of inhibition from both sides is crucial to explain existing data.

Our rigorous re-evaluation also revealed that inhibitory control over excitatory timing is more diverse than originally proposed. 
a

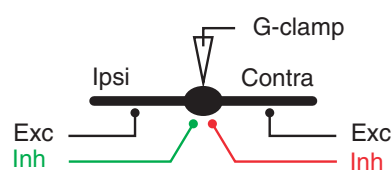

b

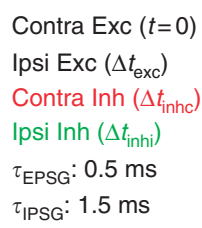

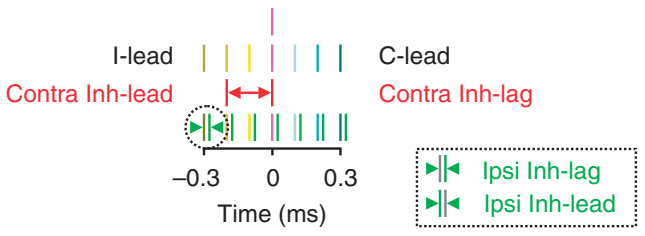

C Contra Inh only Contra + Ipsi Inh
$\Delta t_{\text {inhc }}: 0.2 \mathrm{~ms} \quad \Delta t_{\text {inhi: }}: 0.2 \mathrm{~ms} \quad \Delta t_{\text {inhi: }}=0.1 \mathrm{~ms} \quad \Delta t_{\text {inhi: }}:-0.4 \mathrm{~ms}$

I-lead bias

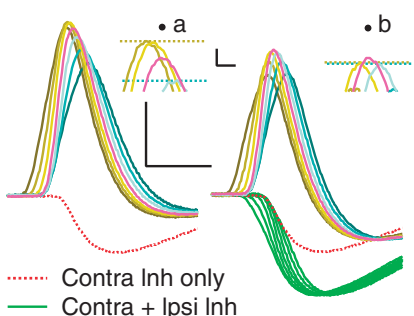

— Contra + Ipsi Inh

f

$\Delta t_{\text {inhc }}:-0.1 \mathrm{~ms} \quad \Delta t_{\text {inhi }}: 0.2 \mathrm{~ms} \quad \Delta t_{\text {inhi: }}-0.1 \mathrm{~ms} \quad \Delta t_{\text {inhi: }}: 0.4 \mathrm{~ms}$

No bias
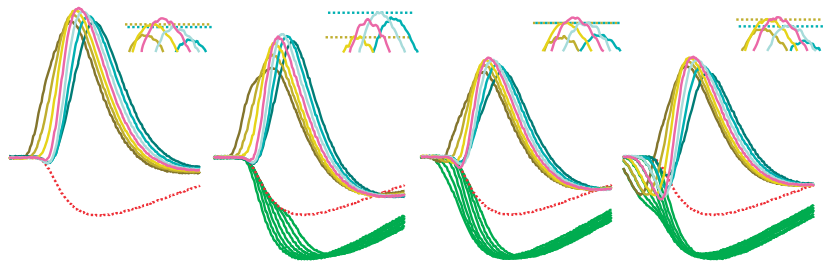

i

$\Delta t_{\text {inhc: }}:-0.4 \mathrm{~ms} \quad \Delta t_{\text {inhi }}: 0.2 \mathrm{~ms} \quad \Delta t_{\text {inhi: }}=0.1 \mathrm{~ms} \quad \Delta t_{\text {inhi: }}=0.4 \mathrm{~ms}$

C-lead bias

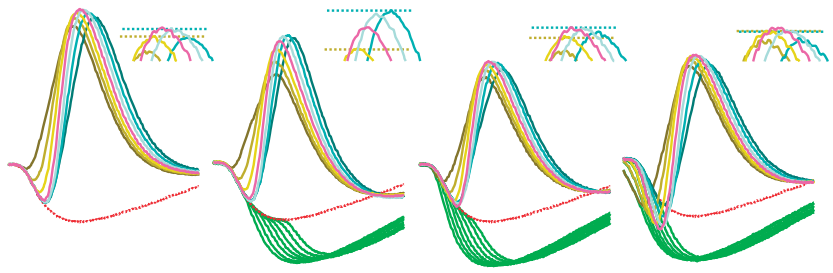

d

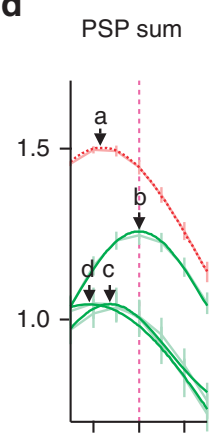

e

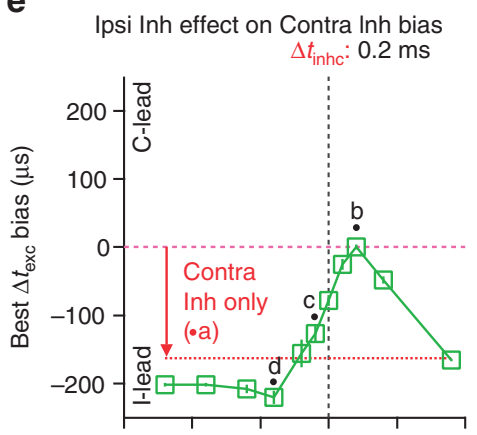

g

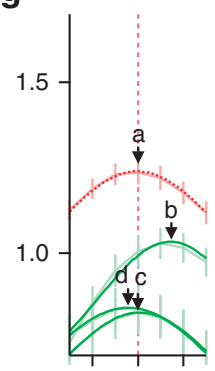

h

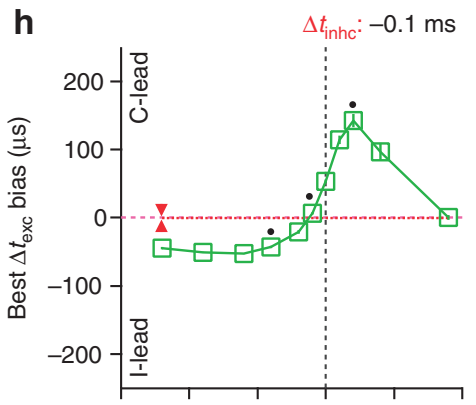

j

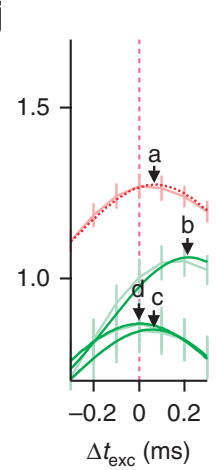

$\mathbf{k}$

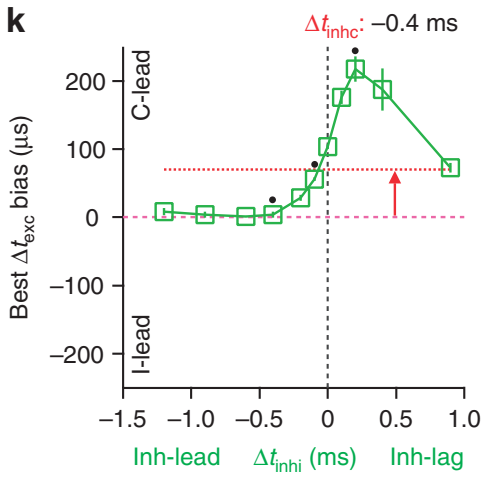

Figure 7 | Bilateral inhibition extends the range of tuning. (a,b) Schematic (a) and timing convention (b) for simulating all four synaptic inputs. The slow-speed EPSG was paired with the average-speed IPSG. Note that ipsilateral Inh is timed relative to Ipsilateral excitation (Exc; $\Delta t_{\text {inhi }}$ ), and the relative timing of contralateral Inh is specified here as $\Delta \mathrm{t}_{\text {inhc. }}$. (c,f,i) Voltage traces for an example recording showing the effect of an Ipsi IPSP on PSP summation with a pre-existing contralateral IPSP (left panels) timed at $\Delta t_{\text {inhc }}$ to bias PSP summation towards Ipsi-leading Exc ( 0.2 ms, c), produce no bias $(-0.1 \mathrm{~ms}, \mathbf{f})$ and bias contralateral-leading Exc $(-0.4 \mathrm{~ms}$, i). Scale bar, $5 \mathrm{mV}, 1 \mathrm{~ms}$. The composite PSP of all four inputs (three right panels) use Ipsi inhibitory timing that matches each of the contralateral inhibitory timing conditions. Composite PSP traces are colour-coded as in Fig. 4, except the contralateral IPSP traces are dotted red, and the composite contralateral and Ipsi IPSP traces are green. Insets are zooms of the peaks, and dotted lines indicate the peaks of flanking timing conditions around zero $\Delta t_{\text {exc }}$ to illustrate bias (as in Fig. $4 \mathrm{~b}, \mathrm{~d}, \mathrm{f}, \mathrm{h}$ ). Letters and dots indicate the timing conditions marked in subsequent analyses. Inset scale bar, $0.5 \mathrm{mV}, 0.1 \mathrm{~ms}$. (d, g,j) PSP summation functions of conditions marked in (c,f,e), respectively, (as in Fig. 4b,d,f,h). (e,h,k) Best $\Delta t_{\text {exc }}$ ( \pm s.d.) plotted against Ipsi inhibitory timing (as in Fig. 4 j,, , but against $\Delta t_{\text {inhi }}$ ) for contralateral inhibitory timing that biased Ipsi-leading Exc $\left(\Delta t_{\text {inhc }}=0.2 \mathrm{~ms}, \mathbf{e}\right)$, produced no bias $\left(\Delta t_{\text {inhc }}=-0.1 \mathrm{~ms}, \mathbf{h}\right)$, and biased contralateral-leading Exc $\left(\Delta t_{\text {inhc }}=-0.4 \mathrm{~ms}, \mathbf{k}\right)$. Dotted red lines and arrows indicate the pre-existing bias enforced by the contralateral Inh alone; $n=7$ recordings. Inh, Inhibition.

For example, even small differences in synaptic kinetics (Fig. 2a-f), the driving force on inhibition (Fig. 2g), the balance of excitation and inhibition (Fig. 2g,h), and the activation of $\mathrm{K}_{\mathrm{LTA}}$ channels (Fig. 3) markedly influenced the efficacy of inhibitionenforced EPSP peak shifts. Moreover, our investigation of a wide range of timing parameters revealed conditions that biased best coincidence detection towards the ipsilateral-leading side (Fig. 4d,e) and others that produced no bias at all (Fig. 4f,g). As the actual arrival time of inhibition relative to excitation in vivo could be adjusted on a single-neuron basis, inhibition- 
a Independent Ipsi and Contra Inh

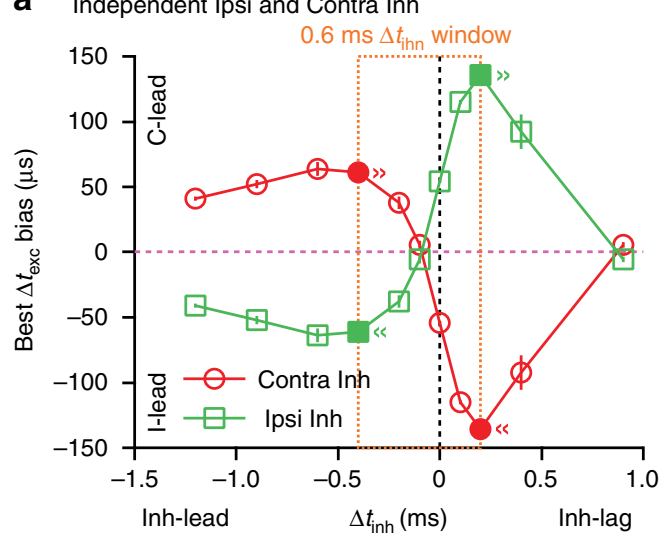

b Combination of Ipsi and Contra Inh

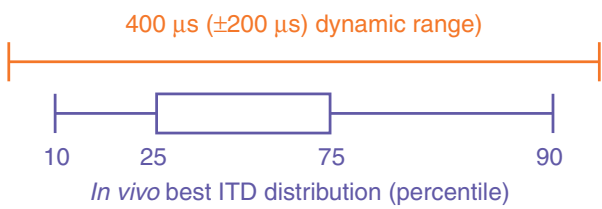

Figure 8 | Bilateral inhibition can explain ITD tuning in vivo. (a) The independent influence of contralateral (Contra) and ipsilateral (Ipsi) Inh on best $\Delta t_{\text {exc }}\left( \pm\right.$ s.d.) plotted against $\Delta t_{\text {inhc }}$ and $\Delta t_{\text {inhir }}$ respectively. For the Contra Inh, data from Fig. $4 \mathrm{k}$ are re-plotted (red circles). To illustrate the near equal and opposite effect of Ipsi Inh on coincidence detection, the data are inverted (green squares). Both Ipsi and Contra Inh can independently influence coincidence detection timing towards the ipsilateral-leading (negative values) and Contra-leading (positive values, light blue shading) sound, depending steeply on their relative arrival timing. The full dynamic range of inhibitory influence on ITD tuning is achieved in a timing window of $0.6 \mathrm{~ms}$ (dotted orange box). Ipsilateral and Contra inhibitory timing conditions that maximally bias ipsilateral-leading $\left(\Delta t_{\text {inhc }}=0.2 \mathrm{~ms}\right.$, $\left.\Delta t_{\text {inhi }}=-0.4 \mathrm{~ms}\right)$ and Contra-leading excitation $\left(\Delta t_{\text {inhc }}=-0.4 \mathrm{~ms}\right.$, $\Delta t_{\text {inhi }}=0.2 \mathrm{~ms}$ ) are filled and marked. (b) Comparison between the dynamic range of bilateral Inh on coincidence detection and the diversity of ITD functions observed in vivo. The dynamic range is calculated by taking the difference between the combinatorial bias of bilateral Inh under inhibitory timing conditions that are marked in a. Although the magnitude of intrinsic delays in the absence of Inh is not known, the range of bilateral inhibitory control of coincidence detection timing could modulate the preferred ITD of a neuron (best ITD) on an individual basis to an extent that exceeds $80 \%$ of the population distribution from previously measured ITD functions in vivo ${ }^{42}$. Inh, Inhibition.

enforced ITD tuning in vivo may be more diverse than the existing data would suggest ${ }^{6,7}$. This provides a powerful mechanism for tuning individual neurons in the MSO, a central issue that remains unexplained by anatomical specializations ${ }^{11,41}$. Although inhibition is one of several mechanisms that may work in concert to tune neurons to their preferred ITDs, it can accomplish this simply by adjustments or adaptations of synaptic properties $^{43,44}$, a more flexible solution compared with axonal length adjustments ${ }^{11}$. Thus, our findings indicate that the original inhibitory model should be expanded.

Recently, one study evaluated the predictions of the original inhibitory model ${ }^{26}$, specifically whether a leading contralateral inhibition biased ITD functions towards the contralateral side. Although there was already evidence that the inhibitory input pathways are faster compared with their excitatory counterpart $^{22,23,45}$, this was directly evaluated in a thick slice preparation by stimulating the auditory nerve. Inhibition indeed preceded excitation, but a single IPSP at the average timing condition $\left(\Delta t_{\mathrm{inh}}=-0.3 \mathrm{~ms}\right)$ did not tune ITD functions. It was concluded that inhibition, while important for coincidence detection, is not relevant for ITD tuning ${ }^{26}$. Although this study used synaptic stimulation instead of conductance-clamp to generate ITD functions, our findings are in accord, as a similar timing condition $\left(\Delta t_{\mathrm{inh}}=-0.2 \mathrm{~ms}\right)$ also did not substantially influence coincidence detection timing (Fig. 4f,g). Importantly, the relative arrival time of excitation and inhibition measured in the study were performed in P15-20 tissue and thus might not represent the fully matured stage of the circuit $^{26}$. Moreover, the measured relative inhibitory timings spanned a range of -0.5 to $-0.1 \mathrm{~ms}^{26}$ and are likely even more diverse in vivo, owing to cochlear delays and natural activation of the cochlear nucleus ${ }^{12,16,46}$ that are lost in a slice preparation. Therefore, we deliberately made no assumptions about the actual arrival times of excitation and inhibition and indeed found other timing conditions that generated pronounced modulation of coincidence detection timing. Finally, the ipsilateral source of inhibition, which appeared equivalently as strong as the contralateral source, was not previously evaluated in modulating coincidence detection $^{26}$. Our present results indicate that the ipsilateral source of inhibition may be in fact crucial to ITD tuning, and thus future studies are required to elucidate the role of its substantial input to the MSO.

The role of synaptic inhibition in the MSO remains a topic of intense debate ${ }^{27-29}$. A second recent study investigated ITD tuning in vivo using whole-cell recordings. A pronounced leading inhibition could not be detected ${ }^{8}$, contradicting the in vitro measurements. It was nevertheless reasoned from the lack of detection that the temporal precision of inhibition was not sufficient to tune ITD functions. In contrast, our findings demonstrate that inhibitory modulation of excitatory timing is in fact resilient to synaptic jitter (Fig. 5) and summation (Fig. 6). More importantly, under timing conditions where inhibition is most effective at influencing coincidence detection timing (Fig. 8a), IPSPs would be masked by EPSPs on each cycle. Even if inhibition could be detected between cycles at low frequencies, its interaction with excitation during highfrequency activity would mask its presence, regardless of its relative timing (Fig. 6a and Supplementary Fig. 8). Unfortunately, as currently available in vivo electrophysiological techniques are insufficient to resolve excitatory and inhibitory events independently ${ }^{8}$, the exact arrival time of excitation and inhibition to MSO neurons remains unknown. The manner in which inhibition tunes ITD functions in vivo thus remains an open question.

The scope of inhibitory mechanisms that modulate ITD coding extends far beyond the precise modulation of coincidence detection timing we report here. For example, the MSO receives GABAergic input, which activates presynaptic $\mathrm{GABA}_{\mathrm{B}}$ receptors on $\mathrm{MSO}$ inputs ${ }^{47,48}$. However, in contrast to phasic glycinergic inhibition, $\mathrm{GABA}_{\mathrm{B}}$ signalling enforces a gain control mechanism to adapt ITD sensitivity in dynamic auditory environments ${ }^{43}$. Furthermore, phasic ${ }^{49,50}$ and sustained ${ }^{51}$ inhibition are also prominent in nucleus laminaris (NL), the functionally equivalent avian analogue to the mammalian $\mathrm{MSO}^{52}$. In contrast to the MSO, inhibition in NL is predonimantly GABAergic and imposes a shunting and depolarizing effect on NL neurons ${ }^{48,49}$. Here phasic and tonic inhibition work in concert to maintain ITD sensitivity at different frequencies and intensities ${ }^{53,54}$. The inhibitory shunt sharpens the coincidence detection time window $^{55}$ in a frequency region-specific manner, but it does not influence the peak timing of incoming excitatory signals ${ }^{50-52}$. Thus, both mammals and birds have evolved diverse inhibitory mechanisms to modulate ITD processing. 


\section{Methods}

Tissue preparation. All experiments were performed in accordance with protocols approved by the Deutsche Tierschutzgesetz. Male and female Mongolian gerbils (Meriones unguiculatus) aged P60-P90 or P17 (Supplementary Fig. 4) were anaesthetized with isoflurane [2-chloro-2-(difluoromethoxy)-1,1,1-trifluoroethane] and decapitated. Brains were removed and placed in a warmed $\left(\sim 35^{\circ} \mathrm{C}\right.$, P60-90) or ice-cold $\left(\sim 4{ }^{\circ} \mathrm{C}, \mathrm{P} 17\right)$ dissecting solution containing the following (in $\mathrm{mM}$ ): 200 sucrose, 25 glucose, $25 \mathrm{NaCl}, 25 \mathrm{NaHCO}_{3}, 3 \mathrm{MgCl}_{2}, 3$ myo-inositol, $2.5 \mathrm{KCl}, 2 \mathrm{Na}$-pyruvate, $1.25 \mathrm{NaH}_{2} \mathrm{PO}_{4}, 0.4$ ascorbic acid and $0.1 \mathrm{CaCl}_{2}(\mathrm{pH} 7.4$ when bubbled with $95 \% \mathrm{O}_{2}$ and $5 \% \mathrm{CO}_{2}$ ). Horizontal $(110 \mu \mathrm{m}$ thick, $\mathrm{P} 60-90)$ or coronal $(250 \mu \mathrm{m}$ thick, P17) slices were made from the brainstem using a VT1200S vibratome (Leica). Slices were then incubated for $30-45 \mathrm{~min}$ at $35^{\circ} \mathrm{C}$ in a perfusion saline containing the following (in $\mathrm{mM}$ ): $125 \mathrm{NaCl}, 25 \mathrm{NaHCO}_{3}, 25$ glucose, 3 myo-inositol, $2.5 \mathrm{KCl}, 2 \mathrm{Na}$-pyruvate, $2 \mathrm{CaCl}_{2}, 1.25 \mathrm{NaH}_{2} \mathrm{PO}_{4}, 1 \mathrm{MgCl}_{2}$ and 0.4 ascorbic acid ( $\mathrm{pH} 7.4$ when bubbled with $95 \% \mathrm{O}_{2}$ and $5 \% \mathrm{CO}_{2}$ ).

Electrophysiology. All experiments were performed in perfused saline $\left(1 \mathrm{ml} \mathrm{min}{ }^{-1}\right.$ and bubbled with $95 \% \mathrm{O}_{2}$ and $5 \% \mathrm{CO}_{2}$ ), except that $\mathrm{CaCl}_{2}$ was reduced to $1.2 \mathrm{mM}$. Recording temperature was measured near the slice and maintained at $35 \pm 1^{\circ} \mathrm{C}$ by an SF-28 in-line heater (Warner Instruments) and PH-1 bath chamber heater (Biomedical Instruments). Tissue was visualized under a BX50WI upright microscope (Olympus) equipped with infrared Dodt Gradient Contrast optics (Luigs \& Neumann), a Polychrome V monochromator (Till Photonics) and an Imago camera (TILL Photonics). Whole-cell recordings of morphologically identified MSO neurons (Fig. 1a) were obtained with borosilicate glass electrodes using an EPC10/2 patch-clamp amplifier (HEKA Elektronik).

Voltage-clamp experiments. For voltage-clamp experiments (Supplementary Fig. 1), the internal solution contained the following (in $\mathrm{mM}$ ): 130 Cs-gluconate, 20 TEA-Cl (tetraethylammonium chloride), 15 HEPES [4-(2-hydroxyethyl)-1piperazineethanesulfonic acid], 5 Cs-EGTA (caesium ethylene glycol tetraacetic acid), 5 QX-314 [N-(2,6-dimethylphenylcarbamoylmethyl) triethylammonium chloride], $5 \mathrm{Na}_{2}$-phosphocreatine, $3 \mathrm{Mg}$-ATP, $0.3 \mathrm{Na}_{2}$-GTP, 0.05 Alexa Fluor 568 and 0.01 ZD 7288 (4-Ethylphenylamino-1,2-dimethyl-6-methylaminopyrimidinium chloride), adjusted to $\mathrm{pH} 7.25$ and $320 \mathrm{~m}$ Osm. Electrodes had 2-3 M $\Omega$ tip resistances, and experiments were performed at $-75 \mathrm{mV}$ (after correcting for an estimated liquid junction potential for this internal solution of $15 \mathrm{mV}$ ). Series resistance was 4-7 M $\Omega$ and was compensated to a residual of 1.5-2 $\mathrm{M} \Omega$ on the amplifier. Experiments were terminated if the uncompensated series resistance changed by $>10 \%$.

Synaptic inputs were stimulated with borosilicate glass electrodes (of 3-4 M $\Omega$ resistance) filled with saline and placed in the vicinity $(50-150 \mu \mathrm{m})$ of the recorded neuron. Ipsilateral and contralateral glutamatergic (excitatory) inputs could be independently activated because they are well segregated to the lateral and medial sides of the MSO, respectively, (Fig. 1b) ${ }^{55}$. As glycinergic (inhibitory) inputs are not well segregated in the slice, the population of inhibitory conductance waveforms likely reflects inputs from both the lateral and medial nucleus of the trapezoid body (lateral and medial nucleus of the trapezoid body ${ }^{56}$. Axons were stimulated with brief $(0.2 \mathrm{~ms}) 10-50 \mathrm{~V}$ bipolar pulses generated by a Model 2100 -isolated pulse stimulator (A-M Systems). The stimulus voltage was adjusted such that putative single fibres were activated, as indicated by all-or-none threshold responses and $<25 \%$ s.d. in amplitude ${ }^{1}$. Conductance values (Supplementary Fig. 1f) were calculated from the measured reversal potential for EPSCs $(5 \mathrm{mV})$ and the calculated Nernst potential of chloride for IPSCs $(-44 \mathrm{mV})$. All voltage-clamp experiments were performed in the presence of $10 \mu \mathrm{M}$ R-CPP $\{3-[(\mathrm{R})-2$ Carboxypiperazin-4-yl]-propyl-1-phosphonic acid] and $10 \mu \mathrm{M}$ SR 95531 [6-Imino3-(4-methoxyphenyl)-1 $(6 \mathrm{H})$-pyridazinebutanoic acid hydrobromide] to block NMDA receptors and $\mathrm{GABA}_{\mathrm{A}}$ receptors, respectively. Glutamatergic and glycinergic inputs were isolated with $0.5 \mu \mathrm{M}$ strychnine and $20 \mu \mathrm{M}$ DNQX (6,7Dinitroquinoxaline-2,3-dione), respectively.

Single-electrode conductance-clamp experiments. For single-electrode conductance-clamp experiments (Figs 1, 2 and 4-7 and Supplementary Figs 2 and $5-8$ ), the internal solution contained the following (in $\mathrm{mM}$ ): $145 \mathrm{~K}$-gluconate, 15 HEPES, $5 \mathrm{Na}_{2}$-phosphocreatine, $3 \mathrm{Mg}$-ATP, $0.3 \mathrm{Na}_{2}$-GTP and 0.05 Alexa Fluor 568 , adjusted to $\mathrm{pH} 7.25$ and $320 \mathrm{~m}$ Osm. Electrodes had 3.5-5 M $\Omega$ tip resistances, and series resistance (4-6 M 2 ) was $100 \%$ balanced on the bridge of the amplifier (Supplementary Fig. 4a). The liquid junction potential was estimated to be $15 \mathrm{mV}$ and compensated online. Selected synaptic conductance waveforms as measured in Supplementary Fig. 1 were delivered to an SM-1 conductance injection amplifier (Cambridge Conductance), which calculates instantaneous current commands $[I(t)]$ by equation $(1)$.

$$
I(t)=G(t)\left[V(t)-E_{\mathrm{rev}}\right]
$$

This calculation was performed independently for excitatory (reversal potential $\left.\left(E_{\text {rev }}\right)=5 \mathrm{mV}\right)$ and inhibitory $\left(E_{\text {rev }}=-90 \mathrm{mV}\right)^{20}$ synaptic conductance waveform templates $(G(t))$, while simultaneously measuring the membrane potential $(V(t))$. Although single inhibitory fibre conductance was roughly twice that of a single excitatory fibre (Supplementary Fig. $1 \mathrm{~b}, \mathrm{f}$ ), previous studies have indicated that there are also about only half the number of inhibitory inputs compared with excitatory inputs, and thus the effective inhibitory and excitatory synaptic strength is balanced ${ }^{1}$. Therefore, for subthreshold experiments EPSGs and IPSGs were injected with a peak conductance of $30 \mathrm{nS}$, yielding an E-I ratio of 1:1 (except for Fig. $2 \mathrm{~h}$, where the IPSG was reduced to $15 \mathrm{nS}$ ). To minimize recording time and maximize the number of parameters that could be tested in a single recording (typically $<10 \mathrm{~min})^{57}$, EPSG and IPSG template pairs were randomized and then injected at $50 \mathrm{~Hz}$. Current test pulses were delivered directly before and after each acquisition epoch to ensure that $I_{\mathrm{h}}$ activity had not substantially changed ${ }^{56}$. Experiments were terminated if membrane input resistance changed by $>1 \mathrm{M} \Omega$.

EPSP peak shifts (Figs 1-3, 5 and 6 and Supplementary Figs 3 and 5-7) were calculated by comparing the peak EPSP time generated by an EPSG plus IPSG injection with that of an EPSG injection alone. Subthreshold PSP summation bias (Fig. 4b,d,f,h, Fig. 7, and Supplementary Fig. 8) was determined by Gaussian fitting of PSP summation functions. PSP summation functions were generated by presenting two identical EPSGs at different relative times $\left(\Delta t_{\mathrm{exc}}\right.$, in $100 \mu \mathrm{s}$ increments). The amplitude of the composite PSP was then normalized to a single EPSP, and the relationship between the normalized composite PSP amplitude and $\Delta t_{\mathrm{exc}}$ (an in vitro representation of an ITD in the absence of any other intrinsic delay mechanisms) was fitted with a Gaussian function. The peak of the function fit was defined as the best $\Delta t_{\mathrm{exc}}$. Each timing condition was repeated six times in each recording, and analysis was performed on the average voltage responses.

For suprathreshold coincidence detection experiments (Fig. 4c,e,g,i), the timing convention was the same as for subthreshold PSP summation experiments, but $\Delta t_{\text {exc }}$ was presented in $20 \mu$ increments. Because action potential conductance thresholds in MSO neurons are extremely sensitive to changes in membrane potential, baseline current (192 $\pm 66 \mathrm{pA}, n=13$ recordings) was injected to maintain the membrane potential at $-65 \mathrm{mV}$. Then two identical EPSGs were injected with a magnitude ( $\tau_{\mathrm{EPSG}}=0.3 \mathrm{~ms}: 43 \pm 2 \mathrm{nS}, n=13$ recordings; $\left.\tau_{\mathrm{EPSG}}=0.5 \mathrm{~ms}: 32 \pm 2 \mathrm{nS}\right)$ that brought the neuron to threshold $\left(G_{\mathrm{t}}\right)$, defined as $50 \%$ action potential probability at $1 \Delta t_{\text {exc }}$. The protocol was then repeated 10 times at $200 \mathrm{pS}(\sim 3 \%)$ above $G_{\mathrm{t}}$, where action potential probability peaked near $100 \%$. Repeating these experiments with the inclusion of the IPSG $\left(60 \mathrm{nS}, \tau_{\mathrm{IPSG}}=1.5 \mathrm{~ms}\right)$ required an adjustment of EPSG magnitude to depolarize the neuron to $G_{\mathrm{t}}$. Adjustments for each $\Delta t_{\text {inh }}$ using the average-speed EPSG $\left(\tau_{\mathrm{EPSG}}=0.3 \mathrm{~ms}\right)$ were as follows: $0.1 \mathrm{~ms}: 50 \pm 1 \mathrm{nS},-0.2 \mathrm{~ms}: 61 \pm 2 \mathrm{nS}$ and $-0.6 \mathrm{~ms}: 48 \pm 1 \mathrm{nS}$. Adjustments for each $\Delta t_{\text {inh }}$ using the slow-speed EPSG $\left(\tau_{\mathrm{EPSG}}=0.5 \mathrm{~ms}\right)$ were as follows: $0.2 \mathrm{~ms}: 51 \pm 2 \mathrm{nS},-0.1 \mathrm{~ms}: 56 \pm 2 \mathrm{nS}$ and $-0.4 \mathrm{~ms}: 50 \pm 3 \mathrm{nS}$. Owing to the many trials for these experiments, only $1-3$ inhibitory timing conditions were performed in each recording. The average action potential probability function from all 10 trials in each recording were then averaged for each inhibitory timing condition ( $n=8-12$ recordings per condition). For statistical analysis, the data were fitted with Gaussian functions as for subthreshold experiments (Fig. 4b,d,f,h).

For jitter experiments (Fig. 5), four EPSGs and IPSGs (10 nS each) were jittered randomly according to the gamma $(\Gamma)$ distribution generated by equation (2).

$$
P(t+a b)=\frac{t^{a-1} e^{\left(-\frac{t}{b}\right)}}{b^{a} \Gamma(a)}
$$

The function asymmetry variable $a$ was 40 to mimic the shape of sound-evoked spiking output of AVCN neurons $s^{34}$, and the function width variable $b$ was 20 , $40,80,120$ and $160 \mu$ s to evaluate different magnitudes of jitter. This generated function half-widths of $80,160,320,480$ and $640 \mu$ s. Iterations of the jittered four inputs were presented 100 times at each inhibitory timing condition in each recording.

For train experiments (Fig. 6 and Supplementary Figs 7 and 8), individual templates were compounded at $200,333,500$ and $800 \mathrm{~Hz}$ for 16 pulses and then injected. Inhibition-enforced EPSP peak shifts (Fig. 6) were calculated relative to the excitation-only peak time for each pulse in the train. Analysis for subthreshold PSP summation bias (Supplementary Fig. 8) was performed as for single-pulse experiments (Figs 4 and 7). Although depression of synaptic currents has been reported ${ }^{1}$, recent observations indicate little if any depression of synaptic potentials during ongoing activity ${ }^{23}$. Therefore, the conductance amplitude was constant for each pulse in the train. This approach thus maximized the effect of summation at higher frequencies. Each timing condition was repeated six times in each recording.

Fibre stimulation experiments. For combined fibre stimulation and conductanceclamp experiments (Fig. 1c,f,h and Supplementary Fig. 2d,e), synaptic conductance was injected as for single-electrode conductance-clamp experiments, except that an extracellular stimulating electrode was placed on the medial fibre tract, and single-fibre stimulation was obtained in current-clamp. Then an EPSG was selected to match the decay kinetics of the stimulated EPSP, typically $\tau_{\mathrm{EPSG}}=0.2$ or $0.3 \mathrm{~ms}$. The strength of the EPSG template was then adjusted to produce an EPSP with equal amplitude to the synaptically evoked EPSP (Supplementary Fig. 2d). Then the IPSG template amplitude was matched to the EPSG template such that the conductance-clamp-simulated EPSP and fibre-stimulated EPSP would have an equivalent influence of the IPSP (E-I ratio $\sim 1: 1)$. Trials were presented at $10 \mathrm{~Hz}$. To eliminate the potential effects of short-term plasticity during the protocol ${ }^{1}$, each 
trial was directly preceded by a 20 pulse, $10 \mathrm{~Hz}$ train such that the amplitude of fibre-stimulated EPSPs was securely at steady state ${ }^{1}$. Each timing condition was repeated 10 times in each recording. In separate experiments, single-fibre EPSPs and IPSPs were also synaptically evoked in current-clamp for a comparison with conductance-clamp-simulated PSPs (Supplementary Fig. 2b,c, blue).

Dual-electrode conductance-clamp experiments. MSO neuron somata were patched sequentially with two electrodes ${ }^{23}$, but otherwise performed as for singleelectrode conductance-clamp experiments. To determine the potential influence of hardware errors introduced by injecting conductance and measuring voltage through the same electrode, synaptic conductance was always injected through the first electrode (E-1), but membrane voltage was measured using the same electrode or using the second (passive) electrode (E-2) and compared (Supplementary Fig. 3). Both directions were evaluated for each recording. Thus, the terms E-1 and E-2 are functionally and not physically defined.

Data acquisition and analysis. Data were acquired at $100 \mathrm{kHz}$. Voltage-clamp data were additionally filtered at $8 \mathrm{kHz}$ using a shallow three-pole Bessel filter in the amplifier. Analysis was performed off-line using Igor Pro (Wavemetrics). Example conductance-clamp traces represent an average of 6-10 trials except for Fig. 4c,e,g,i and Fig. 5c,d, and Supplementary Fig. 5, which show individual raw traces. Example voltage-clamp (Supplementary Fig. 1b) and current-clamp (Supplementary Fig. 2b, blue) traces represent an average of 30 trials. Group averages are presented as mean \pm s.e.m., except for the results of Gaussian fittings, which are presented as peak time $\left(t_{0}\right) \pm$ s.d. Sample sizes for each experiment were at least five per group, which generated normal distributions (determined by Kolmogorov-Smirnov normality tests, $P<0.05$ ). Statistical analysis of the data was determined using two-way ANOVA in Prism software (GraphPad). Dunnett's post hoc tests were performed to determine statistical significance $(P<0.05)$.

Computational model. A conductance-based point-neuron model was generated to explain subthreshold postsynaptic integration (Fig. 3). The dynamics of the membrane voltage $V$ was simulated by current-balance equation (3).

$$
C_{\mathrm{m}} \frac{\mathrm{d} V}{\mathrm{~d} t}=-\left(I_{\mathrm{h}}+I_{\mathrm{K}_{\mathrm{LTA}}}+I_{\text {syn }}+I_{\text {leak }}\right)
$$

$C_{\mathrm{m}}$ denotes the measured individual cell capacitance (24.6 pF on average). Ohmic currents were assumed in equation (4).

$$
I_{x}(V)=g_{x} a_{x}^{m} b_{x}^{n}\left(V-E_{x}\right)
$$

$g_{x}$ denotes the peak conductance, $a_{x}$ and $b_{x}$ were gating functions with cooperativities of $m$ and $n$, respectively, and $E_{x}$ was the reversal potential. The gating functions adhere to first order kinetics(equations 5 and 6 ).

$$
\frac{\mathrm{d} a}{\mathrm{~d} t}=\frac{a_{\infty}-a}{\tau_{a}} \quad \frac{\mathrm{d} b}{\mathrm{~d} t}=\frac{b_{\infty}-b}{\tau_{b}}
$$

$a_{\infty}$ and $b_{\infty}$ are the steady-state activation, and $\tau_{a}$ and $\tau_{b}$ are the voltage-dependent time constants. The leak current $I_{\text {leak }}$ was passive (that is, $m=n=0$ ) with a reversal potential $E_{\text {leak }}=-90 \mathrm{mV}$ and a conductance $G_{\text {leak }}=0.8 \mathrm{pS} \mu \mathrm{m}^{-2}$, estimated from the measured remaining input resistance (on average $280 \mathrm{M} \Omega$ ) at $-90 \mathrm{mV}$ when $\mathrm{K}_{\mathrm{LTA}}$ and $I_{\mathrm{h}}$ channels were blocked with dendrotoxin-k and ZD 7288, respectively. The $\mathrm{K}_{\mathrm{LTA}}$ current was modelled with a reversal potential of $-105 \mathrm{mV}$ (ref. 37), an average half-activation voltage $\left(V_{0.5}\right)$ of $-72.9 \mathrm{mV}$, and an average activation time constant scaling factor $(q)$ of 0.77 . This resulted in kinetics for the $\mathrm{K}_{\mathrm{LTA}}$ channels in equations (7-10) ( $V$ in $\mathrm{mV}, \tau$ in $\mathrm{ms}, m=4, n=1)$.

$$
\begin{gathered}
a_{\infty}(V)=\left(1+e^{\frac{V+92.74}{-11.7}}\right)^{-1} \\
\tau_{a}(V)=q^{-1}\left(\frac{21.5}{6 e^{\frac{V+95.4}{7}}+24 e^{\frac{V+95.4}{50.6}}}\right)+0.35 \\
b_{\infty}(V)=\frac{1-0.27}{1+e^{\frac{V+102.4}{6.16}}}+0.27 \\
\tau_{b}(V)=\frac{170}{5 e^{\frac{V+95.4}{10}}+e^{-\frac{V+105.4}{8}}}+10.7
\end{gathered}
$$

These equations are, apart from the scaling factor $q$, the same as given by Mathews et al. ${ }^{39}$ with $35.4 \mathrm{mV}$ added to all voltages.

The kinetics of the $\mathrm{I}_{\mathrm{h}}$ current were modelled by equations 11 and 12 .

$$
\begin{aligned}
& a_{\infty}(V)=\left(1+e^{0.1536(V+73.97)}\right)^{-1} \\
& \tau_{a}(V)=7 \cdot \frac{28.17+100.9 e^{\frac{-(V+63.2)^{2} / 2}{364.8}}}{3^{(39-32) / 10}}
\end{aligned}
$$

This is similar to the $\mathrm{I}_{\mathrm{h}}$ current of Rothman and Manis ${ }^{58}$, but about three times slower. The cooperativity was set to $m=1$, and the reversal potential was set to $-50 \mathrm{mV}$.

The synaptic conductance templates were the same as those used for conductance-clamp experiments in Fig. $2\left(\tau_{\mathrm{EPSG}}=0.3, \tau_{\mathrm{IPSG}}=1.5 \mathrm{~ms}, n=16\right.$; $\left.\tau_{\mathrm{EPSG}}=0.5 \mathrm{~ms}, n=5\right)$. Conductance-clamp-simulated voltage traces were fitted using the following four parameters: $V_{0.5}$ and $q$ of $\mathrm{K}_{\mathrm{LTA}}$ (Fig. $3 \mathrm{~b}$ ), and the peak conductances of $I_{\mathrm{h}}$ and $\mathrm{K}_{\mathrm{LTA}}$ currents. These parameters were adjusted to fit the cell-specific PSP kinetics, resting potential and input resistance according to conductance-clamp measurements.

\section{References}

1. Couchman, K., Grothe, B. \& Felmy, F. Medial superior olivary neurons receive surprisingly few excitatory and inhibitory inputs with balanced strength and short-term dynamics. J. Neurosci. 30, 17111-17121 (2010).

2. Klumpp, R. G. \& Eady, H. R. Some measurements of interaural time difference thresholds. J. Acoust. Soc. Am. 28, 859-860 (1956).

3. Grothe, B., Pecka, M. \& McAlpine, D. Mechanisms of sound localization in mammals. Physiol. Rev. 90, 983-1012 (2010).

4. Goldberg, J. M. \& Brown, P. B. Response of binaural neurons of dog superior olivary complex to dichotic tonal stimuli: some physiological mechanisms of sound localization. J. Neurophysiol. 32, 613-636 (1969).

5. Yin, T. C. \& Chan, J. C. Interaural time sensitivity in medial superior olive of cat. J. Neurophysiol. 64, 465-488 (1990).

6. Pecka, M., Brand, A., Behrend, O. \& Grothe, B. Interaural time difference processing in the mammalian medial superior olive: the role of glycinergic inhibition. J. Neurosci. 28, 6914-6925 (2008).

7. Brand, A., Behrend, O., Marquardt, T., McAlpine, D. \& Grothe, B. Precise inhibition is essential for microsecond interaural time difference coding. Nature 417, 543-547 (2002).

8. Van der Heijden, M. et al. Directional hearing by linear summation of binaural inputs at the medial superior olive. Neuron 78, 936-948 (2013).

9. Jeffress, L. A. A place theory of sound localization. J. Comp. Physiol. Psychol. 41, 35-39 (1948).

10. McAlpine, D., Jiang, D. \& Palmer, A. R. A neural code for low-frequency sound localization in mammals. Nat. Neurosci. 4, 396-401 (2001).

11. Karino, S., Smith, P. H., Yin, T. C. T. \& Joris, P. X. Axonal branching patterns as sources of delay in the mammalian auditory brainstem: a re-examination. J. Neurosci. 31, 3016-3031 (2011).

12. Van der Heijden, M., Louage, D. H. G. \& Joris, P. X. Responses of auditory nerve and anteroventral cochlear nucleus fibers to broadband and narrowband noise: implications for the sensitivity to interaural delays. J. Assoc. Res. Otolaryngol. 12, 485-502 (2011).

13. Spitzer, M. W. \& Semple, M. N. Neurons sensitive to interaural phase disparity in gerbil superior olive: diverse monaural and temporal response properties. J. Neurophysiol. 73, 1668-1690 (1995).

14. Jercog, P. E., Svirskis, G., Kotak, V. C., Sanes, D. H. \& Rinzel, J. Asymmetric excitatory synaptic dynamics underlie interaural time difference processing in the auditory system. PLoS Biol. 8, e1000406 (2010).

15. Zhou, Y., Carney, L. H. \& Colburn, H. S. A model for interaural time difference sensitivity in the medial superior olive: interaction of excitatory and inhibitory synaptic inputs, channel dynamics, and cellular morphology. J. Neurosci. 25, 3046-3058 (2005).

16. Pena, J. L., Viete, S., Funabiki, K., Saberi, K. \& Konishi, M. Cochlear and neural delays for coincidence detection in owls. J. Neurosci. 21, 9455-9459 (2001).

17. Leibold, C. \& van Hemmen, J. L. Spiking neurons learning phase delays: how mammals may develop auditory time-difference sensitivity. Phys. Rev. Lett. 94, 168102 (2005).

18. McAlpine, D. \& Grothe, B. Sound localization and delay lines-do mammals fit the model? Trends Neurosci. 26, 347-350 (2003).

19. Kadner, A. \& Berrebi, A. S. Encoding of temporal features of auditory stimuli in the medial nucleus of the trapezoid body and superior paraolivary nucleus of the rat. Neuroscience 151, 868-887 (2008).

20. Magnusson, A. K., Kapfer, C., Grothe, B. \& Koch, U. Maturation of glycinergic inhibition in the gerbil medial superior olive after hearing onset. J. Physiol. 568, 497-512 (2005).

21. Chirila, F. V., Rowland, K. C., Thompson, J. M. \& Spirou, G. A. Development of gerbil medial superior olive: integration of temporally delayed excitation and inhibition at physiological temperature. J. Physiol. 584, 167-190 (2007).

22. Grothe, B. \& Sanes, D. H. Bilateral inhibition by glycinergic afferents in the medial superior olive. J. Neurophysiol. 69, 1192-1196 (1993).

23. Grothe, B. \& Sanes, D. H. Synaptic inhibition influences the temporal coding properties of medial superior olivary neurons: an in vitro study. J. Neurosci. 14, 1701-1709 (1994).

24. Leibold, C. Influence of inhibitory synaptic kinetics on the interaural time difference sensitivity in a linear model of binaural coincidence detection. J. Acoust. Soc. Am. 127, 931-942 (2010).

25. Lehnert, S. et al. Action potential generation in an anatomically constrained model of medial superior olive axons. J. Neurosci. 34, 5370-5384 (2014).

26. Roberts, M. T., Seeman, S. C. \& Golding, N. L. A mechanistic understanding of the role of feedforward inhibition in the mammalian sound localization circuitry. Neuron 78, 923-935 (2013).

27. Portfors, C. V. \& von Gersdorff, H. Macrocircuits for sound localization use leaky coincidence detectors and specialized synapses. Neuron 78, 755-757 (2013). 
28. Grothe, B. New roles for synaptic inhibition in sound localization. Nat. Rev. Neurosci. 4, 540-550 (2003).

29. Joris, P. \& Yin, T. C. T. A matter of time: internal delays in binaural processing. Trends Neurosci. 30, 70-78 (2007).

30. Rautenberg, P. L., Grothe, B. \& Felmy, F. Quantification of the three-dimensional morphology of coincidence detector neurons in the medial superior olive of gerbils during late postnatal development. J. Comp. Neurol. 517, 385-396 (2009).

31. Kuwabara, N. \& Zook, J. M. Projections to the medial superior olive from the medial and lateral nuclei of the trapezoid body in rodents and bats. J. Comp. Neurol. 324, 522-538 (1992).

32. Scott, L. L., Mathews, P. J. \& Golding, N. L. Posthearing developmental refinement of temporal processing in principal neurons of the medial superior olive. J. Neurosci. 25, 7887-7895 (2005).

33. Borst, J. G. G. \& Soria van Hoeve, J. The calyx of held synapse: from model synapse to auditory relay. Annu. Rev. Physiol. 74, 199-224 (2012).

34. Joris, P. X., Carney, L. H., Smith, P. H. \& Yin, T. C. Enhancement of neural synchronization in the anteroventral cochlear nucleus. I. Responses to tones at the characteristic frequency. J. Neurophysiol. 71, 1022-1036 (1994).

35. Couchman, K., Grothe, B. \& Felmy, F. Functional localization of neurotransmitter receptors and synaptic inputs to mature neurons of the medial superior olive. J. Neurophysiol. 107, 1186-1198 (2012).

36. Kapfer, C., Seidl, A. H., Schweizer, H. \& Grothe, B. Experience-dependent refinement of inhibitory inputs to auditory coincidence-detector neurons. Nat. Neurosci. 5, 247-253 (2002).

37. Morest, D. K. The collateral system of the medial nucleus of the trapezoid body of the cat, its neuronal architecture and relation to the olivo-cochlear bundle. Brain Res. 9, 288-311 (1968).

38. Mathews, P. J., Jercog, P. E., Rinzel, J., Scott, L. L. \& Golding, N. L. Control of submillisecond synaptic timing in binaural coincidence detectors by $\mathrm{K}(\mathrm{v}) 1$ channels. Nat. Neurosci. 13, 601-609 (2010).

39. Baumann, V. J., Lehnert, S., Leibold, C. \& Koch, U. Tonotopic organization of the hyperpolarization-activated current (Ih) in the mammalian medial superior olive. Front. Neural Circuits 7, 117 (2013).

40. Köppl, C. Phase locking to high frequencies in the auditory nerve and cochlear nucleus magnocellularis of the barn owl, Tyto alba. J. Neurosci. 17, 3312-3321 (1997).

41. Spirou, G. A., Rowland, K. C. \& Berrebi, A. S. Ultrastructure of neurons and large synaptic terminals in the lateral nucleus of the trapezoid body of the cat. J. Comp. Neurol. 398, 257-272 (1998).

42. Smith, P. H., Joris, P. X. \& Yin, T. C. Projections of physiologically characterized spherical bushy cell axons from the cochlear nucleus of the cat: evidence for delay lines to the medial superior olive. J. Comp. Neurol. 331, 245-260 (1993).

43. Stange, A. et al. Adaptation in sound localization: from GABA(B) receptormediated synaptic modulation to perception. Nat. Neurosci. 16, 1840-1847 (2013).

44. Siveke, I., Leibold, C., Schiller, E. \& Grothe, B. Adaptation of binaural processing in the adult brainstem induced by ambient noise. J. Neurosci. 32, 462-473 (2012)

45. Park, T. J., Grothe, B., Pollak, G. D., Schuller, G. \& Koch, U. Neural delays shape selectivity to interaural intensity differences in the lateral superior olive. J. Neurosci. 16, 6554-6566 (1996).

46. Day, M. L. \& Semple, M. N. Frequency-dependent interaural delays in the medial superior olive: implications for interaural cochlear delays. J. Neurophysiol. 106, 1985-1999 (2011).

47. Hassfurth, B., Grothe, B. \& Koch, U. The mammalian interaural time difference detection circuit is differentially controlled by GABAB receptors during development. J. Neurosci. 30, 9715-9727 (2010).

48. Fischl, M. J., Combs, T. D., Klug, A., Grothe, B. \& Burger, R. M. Modulation of synaptic input by GABAB receptors improves coincidence detection for computation of sound location. J. Physiol. 590, 3047-3066 (2012).
49. Hyson, R. L., Reyes, A. D. \& Rubel, E. W. A depolarizing inhibitory response to GABA in brainstem auditory neurons of the chick. Brain Res. 677, 117-126 (1995).

50. Yang, L., Monsivais, P. \& Rubel, E. W. The superior olivary nucleus and its influence on nucleus laminaris: a source of inhibitory feedback for coincidence detection in the avian auditory brainstem. J. Neurosci. 19, 2313-2325 (1999).

51. Tang, Z.-Q., Dinh, E. H., Shi, W. \& Lu, Y. Ambient GABA-activated tonic inhibition sharpens auditory coincidence detection via a depolarizing shunting mechanism. J. Neurosci. 31, 6121-6131 (2011).

52. Köppl, C. Evolution of sound localisation in land vertebrates. Curr. Biol. 19, R635-R639 (2009).

53. Yamada, R. et al. The cooperation of sustained and phasic inhibitions increases the contrast of ITD-tuning in low-frequency neurons of the chick nucleus laminaris. J. Neurosci. 33, 3927-3938 (2013).

54. Tang, Z.-Q. \& Lu, Y. Two GABAA responses with distinct kinetics in a sound localization circuit. J. Physiol. 590, 3787-3805 (2012)

55. Kuba, H., Koyano, K. \& Ohmori, H. Development of membrane conductance improves coincidence detection in the nucleus laminaris of the chicken. J. Physiol. 540, 529-542 (2002).

56. Stotler, W. A. An experimental study of the cells and connections of the superior olivary complex of the cat. J. Comp. Neurol. 98, 401-431 (1953)

57. Khurana, S. et al. An essential role for modulation of hyperpolarizationactivated current in the development of binaural temporal precision. J. Neurosci. 32, 2814-2823 (2012).

58. Rothman, J. S. \& Manis, P. B. Kinetic analyses of three distinct potassium conductances in ventral cochlear nucleus neurons. J. Neurophysiol. 89, 3083-3096 (2003).

\section{Acknowledgements}

We wish to thank Michael Pecka, Nick Lesica and Michael Beierlein for their helpfu comments on the manuscript. This work was supported by the German Science Foundation Collaborative Research Centre (CRC) 870 (to S.L., C.L., F.F. and B.G.), the Alexander von Humboldt Foundation (to M.H.M.), and the Elisabeth and Helmut Uhl Foundation (to F.F.)

\section{Author contributions}

M.H.M. performed the electrophysiology experiments and analysis; S.L. generated the computational model; F.F., C.L. and B.G. provided intellectual guidance; and M.H.M., F.F., C.L. and B.G. wrote the manuscript.

\section{Additional information}

Supplementary Information accompanies this paper at http://www.nature.com/ naturecommunications

Competing financial interests: The authors declare no competing financial interests

Reprints and permission information is available online at http://npg.nature.com/ reprintsandpermissions/

How to cite this article: Myoga, M. H. et al. Glycinergic inhibition tunes coincidence detection in the auditory brainstem. Nat. Commun. 5:3790 doi: 10.1038/ncomms4790 (2014).

(2) This work is licensed under a Creative Commons Attributioncc) NonCommercial-NoDerivs 3.0 Unported License. The images or other third party material in this article are included in the article's Creative Commons license, unless indicated otherwise in the credit line; if the material is not included under the Creative Commons license, users will need to obtain permission from the license holder to reproduce the material. To view a copy of this license, visit http://creativecommons org/licenses/by-nc-nd/3.0/ 